\title{
On the Born-Oppenheimer Expansion for Polyatomic Molecules
}

\author{
M. Klein ${ }^{1}$, A. Martinez ${ }^{2}$, R. Seiler ${ }^{1}$, and X. P. Wang ${ }^{1, \star}$ \\ 1 Fachbereich Mathematik MA 7-2, Technische Universität Berlin, W-1000 Berlin 12, \\ Federal Republic of Germany \\ 2 Université Paris-Nord C.S.P., Département de Mathématiques et d'Informatique, \\ Avenue Jean-Baptiste Clément, F-93430 Villetaneuse, France
}

Received July 25, 1991

\begin{abstract}
We consider the Schrödinger operator $P(h)$ for a polyatomic molecule in the semiclassical limit where the mass ratio $h^{2}$ of electronic to nuclear mass tends to zero. We obtain WKB-type expansions of eigenvalues and eigenfunctions of $P(h)$ to all orders in $h$. This allows to treat the splitting of the ground state energy of a non-planar molecule. Our class of potentials covers the physical case of the Coulomb interaction. We use methods of $h$-pseudodifferential operators with operator valued symbols, which by use of appropriate coordinate changes in local coordinate patches covering the classically accessible region become applicable even to our class of singular potentials.
\end{abstract}

\section{Introduction}

Molecular systems are described by the many body Hamiltonian

$$
\begin{gathered}
P(h):=-h^{2} \Delta_{x}+h^{2} p\left(\partial_{y}\right)+Q(x), \\
Q(x)=-\Delta_{y}+V(x, y)+W(x),
\end{gathered}
$$

where $x \in \mathbb{R}^{n}$ denotes the nuclear and $y \in \mathbb{R}^{p}$ the electronic coordinate; $p\left(\partial_{y}\right)$ stands for the isotopic term and $Q$ is the electronic Hamiltonian. It is formally $P(h=0)$. The potential $V$ denotes the electron-electron and nuclei-electron interaction and the potential $W$ the nuclei-nuclei interaction. They are typically of Coulomb type. $h^{2}$ stands for the ratio of electronic and nuclear mass. The isotopic term $p\left(\partial_{y}\right)$ is of second order in $\partial_{y}$ and a result of the non-canonical coordinate system traditionally used in this context: The so-called center of mass of the nuclei system. It is not of the Jacobi type. Figure 1 shows the coordinates used for the case of 2 nuclei $A, B$ and 3 electrons $a, b, c$.CNM stands for center of mass of the nuclei and $y=\left(y_{1}, y_{2}, y_{3}\right)$.

\footnotetext{
* Permanent address: Department of Mathematics, Peking University, 100871 Beijing, China
} 


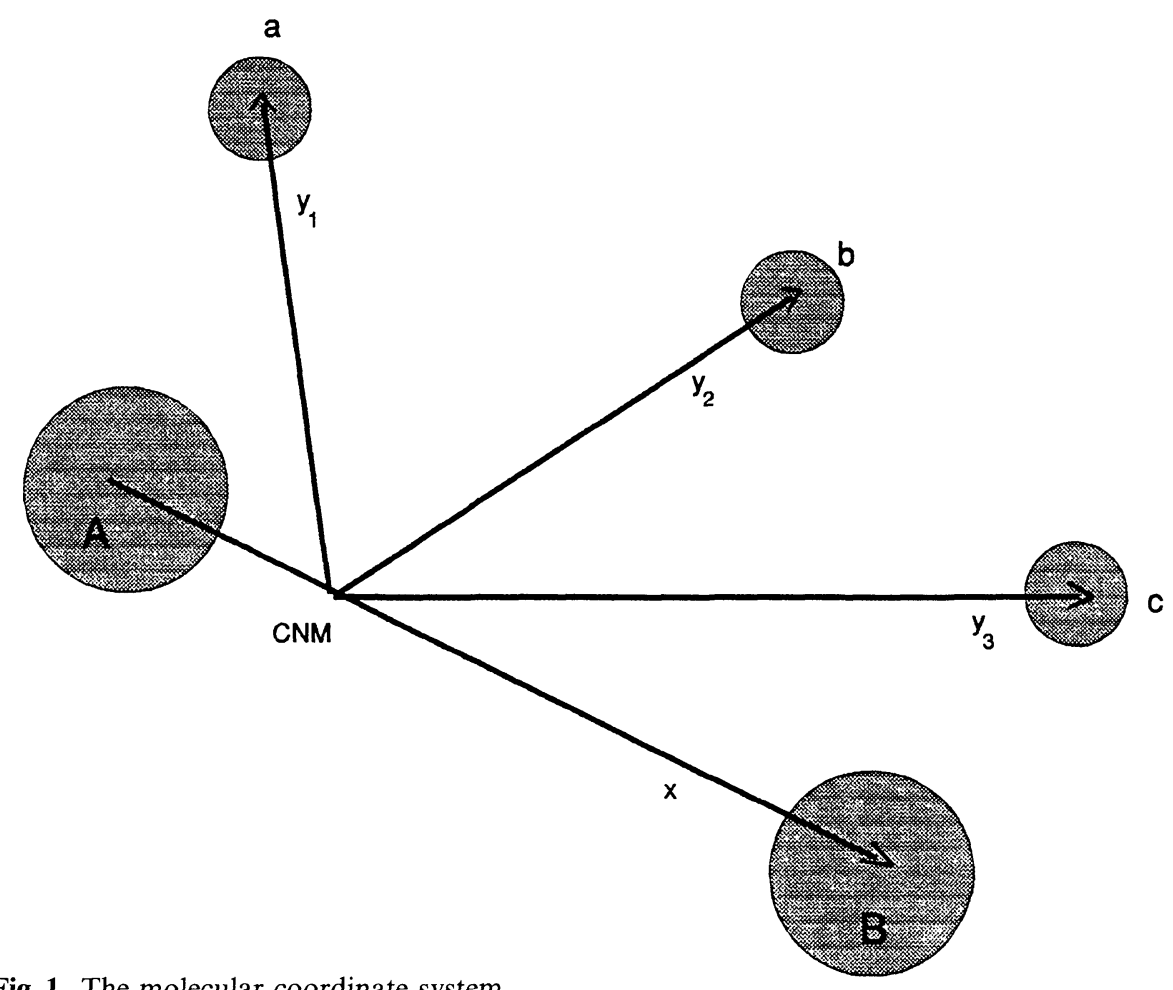

Fig. 1. The molecular coordinate system

The electronic Hamiltonian as well as the isotopic term commute with the nuclear position operator $x$. This makes it possible to introduce the following geometrical picture: Think of the molecular state space $\mathscr{H}$ as the space of square integrable sections in the trivial fiber bundle $\mathbb{R}_{x}^{n} \times L^{2}\left(\mathbb{R}_{y}^{p}\right)$. In this picture the operator $P(h)$ decomposes into two terms. The first one (the nuclear kinetic energy) acts in the base space. The second one (the isotopic term and the electronic Hamiltonian) operates on the fiber only,

$$
Q=\int_{\oplus} d x Q(x) .
$$

$Q(x)$ can be interpreted as the Hamiltonian for the electrons in the external field of the nuclei positioned at $x \in \mathbb{R}_{x}^{n}$. Its spectrum is typically discrete in the low energy region and continuous above a threshold energy.

In molecular Hamiltonians there is a natural small and dimensionless parameter: the mass ratio $h^{2}$. It is typically of the order $10^{-4}$. This suggests a perturbative computation of physically interesting quantities like the spectrum of $P(h)$ or scattering cross sections.

The main challenge in molecular physics in the twenties was to explain the phenomenologically observed 3 scales in the spectra. Born and Oppenheimer after a futile attempt by Born and Heisenberg in the area before the Schrödinger equation - solved the problem. In their seminal paper of 1927 [BO] they developed a formal perturbation theory in $k=\sqrt{h}$ and argued that the zeroth, second and fourth order explains the qualitative picture of molecular spectra. 


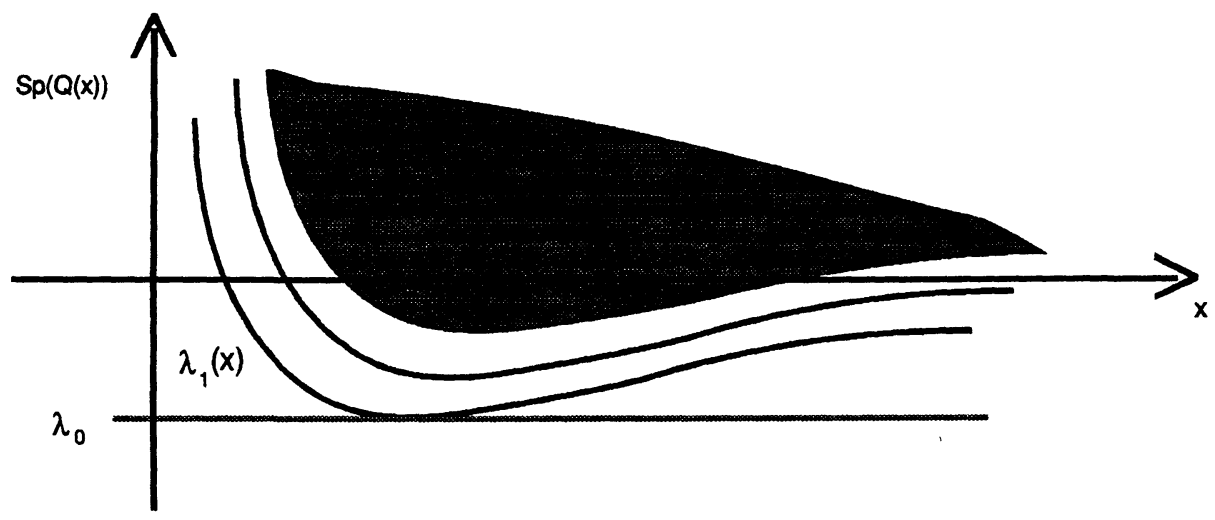

Fig. 2. Discrete and essential spectrum of $Q(x)$

A mathematical justification of the Born-Oppenheimer method started to develop much later. In 1981 the diatomic case with rotationally invariant potentials could be treated using methods of functional analysis [CDS]. Some years later, a discussion of the full asymptotic expansion in $h$ was given by Hagedorn [Ha1, Ha2], using separation of variables and the method of multiple scales. This was generalized to the case of many nuclei for smooth potentials only [Ma2], where WKB-type expansions were given for non-radially symmetric potentials supposed to admit nondegenerate point wells of the effective potential. In this article we shall justify the Born-Oppenheimer expansion for polyatomic molecules and a class of potentials which includes the physically interesting case of Coulomb interactions. The method relies in an essential manner on a technique introduced by Hunziker [Hu]. Hence, in some way, this work is a realization of Remark 6 in the article by Hagedorn [Ha2].

The mathematical analysis of the Born-Oppenheimer method faces two main obstacles which we want to describe now in general terms. They are related to the semiclassical nature of the problem and in part to the main technical tool for treating such problems: the pseudodifferential calculus.

In the classical setting the operator to be discussed is of the type $-h^{2} \Delta+V$, where $V$ is the multiplication operator by a smooth potential function. In the molecular case the corresponding object is $Q$. Yet $Q$ is neither a multiplication operator nor smooth (the latter only for non-smooth potentials).

Since $Q$ is really an operator and not just a potential function it becomes necessary to use a pseudodifferential operator calculus with operator valued symbols. Furthermore for the application of pseudodifferential operator techniques, it is useful to translate the eigenvalue problem

$$
P(h) \psi(h)=\lambda(h) \psi(h)
$$

by Grushin's method into the problem of inverting a $2 \times 2$ matrix operator.

Let us describe the structure of this method in slightly more detail. Let $\Pi(x)$ be the spectral projection of $Q(x)$ projecting onto the lowest $N$ eigenvectors. Hence

$$
\Pi=\int_{\oplus} d x \Pi(x)
$$

is a projection on the molecular state space $\mathscr{H} . \Pi$ is a natural object in the context of the semiclassical limit since it is expected (and in certain cases even proved) that 
the spectrum and eigenfunctions of $P$ are well approximated by the spectrum and eigenfunctions of ПРП.

The Grushin operator is defined by

$$
\mathscr{P}_{\lambda}=\left[\begin{array}{cc}
P-\lambda & i \\
\Pi & 0
\end{array}\right]
$$

and acts on $\mathscr{H} \oplus \Pi \mathscr{H} . i$ denotes the immersion $\Pi \mathscr{H} \rightarrow \mathscr{H}$. Its inverse is formally given by

$$
\mathscr{P}_{\lambda}^{-1}=\left[\begin{array}{cc}
\hat{R}(\lambda) & \Pi-\hat{R}(\lambda) \hat{\Pi} P \Pi \\
\Pi-\Pi P \hat{R}(\lambda) & \lambda-F(\lambda)
\end{array}\right],
$$

where $\hat{R}(\lambda)$ denotes the restriction of the resolvent $\hat{\Pi}(P-\lambda)^{-1}$ to the range of $\hat{\Pi}=1-\Pi . F(\lambda)$ is the Feshbach operator and defined by

$$
F(\lambda)=\Pi P \Pi-\Pi P \hat{R}(\lambda) \hat{\Pi} P \Pi \text {. }
$$

On a formal level it is easily seen that the eigenvalue problem $(0.3)$ is equivalent to the generalized eigenvalue problem for $F(\lambda)$,

$$
F(\lambda) \tilde{\psi}=\lambda \tilde{\psi} .
$$

This program can be implemented in the framework of pseudodifferential operators under appropriate conditions. In particular it is important that $\Pi$ defines a continuous trivial vector bundle over $\mathbb{R}_{x}^{n}$ (or at least over a convenient open subset $\Omega$ ), which is smooth in $x$ after conjugation by a unitary which preserves the fiber structure. More explicitly, there exist global continuous sections $\psi_{i}(x), i=1, \ldots, N$ such that

$$
\Pi(x) \varphi(x)=\sum_{i=1}^{N} \psi_{i}(x)\left\langle\psi_{i}(x), \varphi(x)\right\rangle_{y},
$$

where the scalar product refers to the Hilbert space structure in the fiber. The local smoothness refers to the existence of unitary operators $U(x)$, defined locally in $x$, acting on the fiber over $x$ such that

$$
\tilde{\psi}_{i}(x):=U(x) \psi_{i}(x)
$$

is smooth. This can be summarized by saying: There exists a differentiable structure on the trivial continuous vector bundle defined by $\Pi$ which turns it into a smooth bundle. The origin of this structure is explained in the following paragraph.

The second obstacle mentioned previously is the non-smoothness of $Q$. Let us explain this looking at the following example. The electronic operator for an electron in the field of two nuclei acting in the fiber $x \in \mathbb{R}_{x}^{n}$ is given by

$$
Q(x)=-\Delta_{y}-\frac{1}{|x / 2-y|}-\frac{1}{|x / 2+y|}+\frac{1}{|x|} .
$$

The singularity in $y$ is $x$-dependent. Hence differentiation of $Q(x)$ gives rise to singularities in $y$ which are getting worse with increasing $|\alpha|$ :

$$
D_{x}^{\alpha} Q(x) \sim \frac{1}{|x / 2-y|^{1+|\alpha|}}+\frac{1}{|x / 2+y|^{1+|\alpha|}}+\frac{1}{|x|^{1+|\alpha|}} .
$$


In the diatomic case with rotationally invariant potentials this problem could be removed by a simple dilation technique [CDS]. In the general case this is much more subtle. It can however be overcome by a technique introduced by Hunziker $[\mathrm{Hu}]$ and is similar in spirit to the method used in the diatomic case: In each coordinate patch $\omega \subset \mathbb{R}_{x}^{n}, P(h)$ is replaced by a unitarily conjugated operator, where the unitary $U(x)$ implements a diffeomorphism in $y$ space so that the singularities get $x$-independent. For geometrical reasons this diffeomorphism can only be constructed locally in $x$-space. So a cutting and pasting procedure is needed.

Finally one heuristic word about the singularity introduced by the nuclearnuclear interaction $\frac{1}{|x|}$ in the electronic Hamiltonian $Q(x)$. Its presence is rather harmless because of its repulsive nature (positivity). In the limit $h \downarrow 0$ the wave functions are localized in $x$-space around minima of inf $S p(Q(x))$. So they avoid any point of nuclear collision.

Let us now come to an exposition of the structure of this article. In the first section a framework is developed which is large enough to incorporate physically interesting Hamiltonians. The main goal of this section is the construction of the inverse of the Grushin $N \times N$ matrix operator as a pseudodifferential operator for all $\lambda$ in a certain energy range $I$. The energy range which is considered determines the number $N$. The potentials are assumed to be Kato small, and $W$ must be positive as in the example just mentioned (Hypothesis 1). The function $\lambda_{1}(x)$ $=$ inf $S p(Q(x))$ plays the role of an effective potential function which defines a decomposition of $\mathbb{R}_{x}^{n}$ into a classically allowed region $\Omega$ and the classically forbidden region $\mathbb{R}^{n} \backslash \Omega$ (depending of course on the energy range $I=[-\infty, b$ ) considered, $\lambda^{-1}(I)(\Omega)$. Since most of the interesting phenomena happen in the classically accessible region the relevant assumptions concern $\Omega$ only. In particular, for every $x$ in a neighborhood of $\Omega$ a diffeomorphism $F_{x}: \mathbb{R}_{y}^{p} \rightarrow \mathbb{R}_{y}^{p}$ (locally in $x$ ) is defined such that $V(x, \cdot)$ is smooth in $x$ as a map from $H^{2}\left(\mathbb{R}_{y}^{p}\right)$ to $L^{2}\left(\mathbb{R}_{y}^{p}\right)$ after conjugation by $F$ (Hypothesis 2). In Hypothesis 3 an assumption is introduced which makes the reduction to the $N \times N$ Grushin matrix operator possible.

As mentioned previously, to realize the Grushin operator as a pseudodifferential operator on a manifold, it is crucial to have the triviality of the vector bundle defined by $\Pi$, at least over the classically accessible region. This is ensured by Hypothesis 4. Furthermore, since eigenfunctions are localized in the classically allowed region, it is possible to replace the Grushin operator in the classically forbidden region by a smooth one modulo a small controllable error. In fact we replace $Q$ by an operator $Q^{\zeta}$, which differs from $Q$ in the classically forbidden part of $\mathbb{R}_{x}^{n}$ only. By an argument involving the Agmon inequalities for eigenfunctions of $P$ (see [A], and [HS1] for the semiclassical version) one can show that the substitution of $Q$ by $Q^{\zeta}$ does not change eigenvalues up to an exponentially small term in $h$. It leads to the main result, the existence of the inverse of the Grushin operator as a pseudodifferential operator (Theorem 1.2). As an application (Proposition 1.5) it is shown that the operator $P$ and the operator $\Pi P \Pi$ have the same spectrum in a vicinity of $\lambda_{0}=\inf S p(Q(x))$ of typical size $h$ up to a correction term of order $h^{5 / 2}$.

In the second section it is shown that for molecular Hamiltonians the assumptions of the first chapter are met. So the results can be applied. The eigenvalue problem for $P$ can be replaced by the generalized one for the Feshbach operator. 
In the third section the diatomic case is considered. It is shown that the first $N$ eigenfunctions possess asymptotic expansions in $h$ of the WKB type.

In the fourth chapter the corresponding results are derived for a polyatomic molecule in the ground state. A non-planar polyatomic molecule is shown to display the Jahn-Teller effect, i.e. the minimal set of $\lambda_{1}(x)$ in $\mathbb{R}_{x}^{n}$ is disconnected. The situation is therefore very close to a multiple well problem. In the last section the eigenvalue splitting for this generalized multiple well problem is computed along the lines of the article by Helffer and Sjöstrand [HS1].

\section{Reduction to an Effective Hamiltonian}

We consider the Schrödinger operator

$$
P=-h^{2} \Delta_{x}+h^{2} p\left(\partial_{y}\right)+Q(x) ; \quad Q(x)=-\Delta_{y}+V(x, y)+W(x)
$$

on $L^{2}\left(\mathbb{R}_{x}^{n} \times \mathbb{R}_{y}^{p}\right)$, where $p\left(\partial_{y}\right)$ is a symmetric differential operator of degree 2 with real coefficients and the potentials $V(x, y), W(x)$ satisfy hypothesis $(\mathrm{H} 1),(\mathrm{H} 2)$ below.

In the physical application we have in mind we think of $x \in \mathbb{R}^{n}$ as Jacobi coordinates for the position of $(m+1)$ nuclei in $\mathbb{R}^{3}$ with the center of mass of all nuclei and electrons removed and of $y \in \mathbb{R}^{p}$ as representing the configuration of $q$ electrons (measured from the center of mass of the nuclei alone). We shall see in Sect. 2 that a typical molecular Schrödinger operator is of the form (1.1), where $V(x, y)$ contains the nucleon-electron and the electron-electron interaction, and $W(x)$ contains the nucleon-nucleon interaction.

In this section we shall show that, although $V$ and $W$ in general possess singularities, complete information on the low lying part of the spectrum $S p(P)$ is contained in a certain $h$-admissible (i.e. pseudodifferential up to $O\left(h^{\infty}\right)$ ) operator $E_{-+}(\lambda)$, which is defined by constructing an inverse of an appropriate Grushin problem for $P$. It is essential for this result that the nuclei-nuclei interaction $W$ is repulsive, and that after a suitable $x$-dependent change of coordinates the real multiplication operator $V(x, y)$ depends smoothly on $x$ as an operator with fixed singularities. More precisely, we assume (H1) and (H2) below.

(H1) For all $x \in \mathbb{R}^{n} V(x, \cdot)$ is $\Delta_{y}$-bounded with relative bound $a<1$, uniformly in $x$, and $W \geqq 0$ is $\Delta_{x}$-bounded with relative bound zero.

In particular, for $h$ sufficiently small $P$ is realized as a selfadjoint operator in $L^{2}\left(\mathbb{R}^{n+p}\right)$ with domain $\mathscr{D}(P)=H^{2}\left(\mathbb{R}^{n+p}\right)$ which satisfies $P \geqq-\gamma$ for some $\gamma>0$ uniformly in $h$.

Remark 1.1. We assume $W$ to be $\Delta_{x}$-bounded with relative bound zero only to avoid technical domain questions unrelated to the problems considered here. In fact, since $W \geqq 0$, we could roughly speaking treat any positive singularities of $W$ compatible with selfadjointness as long as $W$ is smooth in a region $\Omega$ satisfying (H3).

(H2) There exists a finite family of open sets $\left(\Omega_{j}\right)_{j=1}^{r}$ in $\mathbb{R}^{n}$ with compact closure and an associated family of mappings $F_{j} \in C^{\infty}\left(\Omega_{j} \times \mathbb{R}^{p} ; \mathbb{R}^{p}\right)$ such that for all $x \in \Omega_{j}, 1 \leqq j \leqq r, F_{j}(x, \cdot)$ is a diffeomorphism of $\mathbb{R}^{p}$, which equals the identity for $|y|$ sufficiently large and satisfies

$$
V\left(x, F_{j}(x, y)\right)\left(-\Delta_{y}+1\right)^{-1} \in C^{\infty}\left(\Omega_{j}, \mathscr{L}\left(L^{2}\left(\mathbb{R}^{p}\right)\right)\right) .
$$


Furthermore

$$
W(x) \in C^{\infty}\left(\Omega_{j}, \mathbb{R}\right) .
$$

Next, for any $M$, we shall denote by $\lambda_{1}(x) \leqq \ldots \leqq \lambda_{M}(x)$ the $M$ first values (not necessarily eigenvalues) given by the Mini-Max principle for the semibounded operator $Q(x)$ on $L^{2}\left(\mathbb{R}_{y}^{p}\right)$, i.e. its first $M$ eigenvalues or possibly $\operatorname{Inf} S p_{\text {ess }}(Q(x))$. Due to possible positive singularities of $W$ outside $\cup \Omega_{j}$ we allow $\infty$ as a value of $\lambda_{j}$. By adding a constant we may assume lim-inf $\lambda_{1}(x)=0$. We are interested in the $|x| \rightarrow \infty$ spectrum of $P$ in an interval $I=(-\infty, b]$ with $\lambda_{0}=\operatorname{Inf}_{\mathbb{R}^{n}} \lambda_{1}<b<0$, and we assume (H3) There exists an open set $\Omega \subset \subset \bigcup_{j=1}^{r} \Omega_{j}$ such that $\lambda_{1}^{-1}(I) \subset \subset \Omega$ and $\mathrm{b}<\operatorname{Inf}_{\Omega} S p(Q(x)) \backslash\left\{\lambda_{1}(x), \ldots, \lambda_{M}(x)\right\}$ for some $M \in \mathbb{N}$.

To study just a small neighborhood of $\lambda_{0}$ it suffices to take $M=1$; however in order to study a larger range $I$ of energy we shall as well treat the case of several electronic levels $\lambda_{j}(x)$. Note, however, that for $M=1$ the groundstate $u_{1}(x, \cdot)$ of $Q(x)$ can be chosen positive for $x \in \Omega$; thus it is globally defined as a continuous function of $x \in \Omega$. Since for $M>1$ this may fail for the eigenfunctions of $Q(x)$ associated with $\lambda_{j}(x), j>1$, we shall in addition assume

(H4) There exists a contractible open set $\widetilde{\Omega}$ containing $\bigcup_{j=1}^{r} \Omega_{j}$ such that for $x \in \tilde{\Omega}$ the $M$ real numbers $\tilde{\lambda}_{i}(x)=\lambda_{i}(x)-W(x), 1 \leqq i \leqq M$, are discrete eigenvalues of $Q(x)-W(x)$, which are separated by a gap from the rest of its spectrum. Furthermore the orthogonal projection $\Pi(x)$ on the associated eigenspace belongs to $C\left(\widetilde{\Omega}, \mathscr{L}\left(L^{2}\left(\mathbb{R}_{y}^{p}\right)\right)\right)$.

We remark that if $P$ is a molecular Schrödinger operator as in $(H$ mol) of Sect. 2 , the projection $\Pi(x)$ is automatically $C^{2}$ in $x$ as long as the gap condition in (H4) is satisfied (see [CS]). Furthermore, by standard arguments it follows from $(\mathrm{H} 2)$ that

$$
U_{j}(x) \Pi(x) U_{j}^{-1}(x) \in C^{\infty}\left(\Omega_{j}, \mathscr{L}\left(L^{2}\left(\mathbb{R}_{y}^{p}\right), H^{2}\left(\mathbb{R}_{y}^{p}\right)\right)\right),
$$

where $U_{j}(x)$ denotes the unitary transformation

$$
\left(U_{j}(x) \varphi\right)(y)=\varphi\left(F_{j}(x, y)\right)\left|\operatorname{det} \partial_{y} F_{j}(x, y)\right|^{1 / 2}
$$

induced by $F_{j}(x, \cdot)$ on $L^{2}\left(\mathbb{R}_{y}^{p}\right)$ for $x \in \Omega_{j}$. In fact, setting $\mathrm{Q}_{j}(x)=U_{j}(x) Q(x) U_{j}^{-1}(x)$, showing (1.4) reduces to proving existence of

$$
\left[Q_{j}(x)-\lambda\right]^{-1} \partial_{x}^{\alpha} Q_{j}(x)\left[Q_{j}(x)-\lambda\right]^{-1} \in \mathscr{L}\left(L^{2}\left(\mathbb{R}_{y}^{p}\right), H^{2}\left(\mathbb{R}_{y}^{p}\right)\right)
$$

for $x \in \Omega_{j}, \alpha \in \mathbb{N}^{n}$ and $\lambda$ in the resolvent set of $Q(x)$. This follows from (H2).

Since in view of $(\mathrm{H} 2) U_{j}(x)$ is bounded in $H^{2}\left(\mathbb{R}_{y}^{p}\right)$, we get $\left[Q_{j}(x)-\lambda\right]^{-1} \in \mathscr{L}\left(L^{2}\left(\mathbb{R}_{y}^{p}\right), H^{2}\left(\mathbb{R}_{y}^{p}\right)\right)$, and using (1.2) in $(\mathrm{H} 2),(1.6)$ can be read off the explicit formula

$$
Q_{j}(x)=-\left(\left(\partial_{y} G_{j}\right)^{T}\left(x, F_{j}(x, y)\right) \partial_{y}+H(x, y)\right)^{2}+V\left(x, F_{j}(x, y)\right)+W(x),
$$

where $G_{j}(x, \cdot)$ denotes the inverse diffeomorphism of $F_{j}(x, \cdot), T$ stands for the transposition, and

$$
H(x, y)=\left|\operatorname{det} \partial_{y} F_{j}\right|^{1 / 2} \partial_{y}\left|\operatorname{det} \partial_{y^{\prime}} G_{j}\left(x, y^{\prime}=F(x, y)\right)\right|^{1 / 2} .
$$


The main point of $(\mathrm{H} 4)$ is to assume $\widetilde{\Omega}$ contractible (which a priori could be violated by eigenvalue crossing and absorption of eigenvalues into the essential spectrum of $Q(x)-W(x))$.

Due to the homotopy property of vector bundles ([BT, Hus]) this ensures that the vector bundle $E \subset \Omega \times L^{2}\left(\mathbb{R}_{y}^{p}\right)$ over $\widetilde{\Omega}$ defined by $\Pi(x)$ is trivial. More precisely, we have

Lemma 1.1. Assume $\mathrm{H} 1-\mathrm{H} 4$. Then there exist $M$ functions $u_{l}(x, y) \in C\left(\widetilde{\Omega}, H^{2}\left(\mathbb{R}_{y}^{p}\right)\right)$, $l=1, \ldots, M$, such that $\forall x \in \bigcup_{j=1}^{r} \Omega_{j}$

$$
\Pi(x)=\sum_{l=1}^{M}\left\langle\cdot, u_{l}(x)\right\rangle_{y} u_{l}(x) ; \quad\left\langle u_{l}(x), u_{k}(x)\right\rangle_{y}=\delta_{l k}
$$

where $\langle\cdot, \cdot\rangle_{y}$ denotes the scalar product in $L^{2}\left(\mathbb{R}_{y}^{p}\right)$, and in addition the sections $u_{l}(x)$ of $E$ become smooth after the coordinate change induced by $F_{j}$, i.e.

$$
U_{j}(x) u_{l}(x, \cdot) \in C^{\infty}\left(\Omega_{j}, H^{2}\left(\mathbb{R}_{y}^{p}\right)\right) \quad(1 \leqq l \leqq M, 1 \leqq j \leqq r) .
$$

Furthermore, for all $x \in \mathbb{R}^{n}$, there is an orthonormal set (with respect to the $L^{2}$-scalar-product) of functions $w_{1}(x), \ldots, w_{M}(x)$ in $H^{2}\left(\mathbb{R}_{y}^{p}\right)$ which coincides with the $u_{l}(x)$ for $x \in \lambda_{1}^{-1}(I) \subset \subset C$, satisfies (1.9) and

$$
w_{l}(x, \cdot) \in C^{\infty}\left(\mathbb{R}^{n} \backslash \Omega, H^{2}\left(\mathbb{R}_{y}^{p}\right)\right) .
$$

Proof. The triviality of the vector bundle $E$ induced by $\Pi$ over $\widetilde{\Omega}$ is equivalent to the existence of $M$ continuous sections $\tilde{u}_{l}(x)$ of $E$ which span the fiber Ran $\Pi(x)$ at each $x \in \widetilde{\Omega}$. Taking $\chi \in C_{0}^{\infty}\left(\left\{z \in \mathbb{R}^{n+p},|z|<1\right\}\right)$ with $\int \chi(z) d z=1$ we introduce the mollifier

$$
M_{\varepsilon} f(z)=\int_{\mathbb{R}^{n+p}} \varepsilon^{-n-p} \chi\left(\frac{z-\tilde{z}}{\varepsilon}\right) f(\tilde{z}) d \tilde{z} .
$$

Setting $H=H^{2}\left(\mathbb{R}_{y}^{p}\right)$ and letting $\Lambda \subset \mathbb{R}^{n}$ be a bounded open set, we claim that for $f \in C_{0}(\Lambda, H)$

$$
\left\|M_{\varepsilon} f-f\right\|_{L^{\infty}(\Lambda, H)} \rightarrow 0 \quad(\varepsilon \downarrow 0) .
$$

In fact it follows from Young's inequality that

$$
\left\|M_{\varepsilon}\right\|_{L^{\infty}\left(\mathbb{R}^{n}, H\right), L^{\infty}\left(\mathbb{R}^{n}, H\right)} \leqq 1 \quad(\varepsilon>0) ;
$$

thus, in view of the Sobolev imbedding theorem and $H_{0}^{s}(\Lambda, H) \cap C_{0}(\Lambda, H)$ being dense in $C_{0}(\Lambda, H)$ for $s>\frac{n}{2}$, it suffices to show (1.11) for $f \in H_{0}^{s}(\Lambda, H)$ with convergence in $\|\cdot\|_{H^{s}(\Lambda, H)}$. For $s$ integer this is an immediate consequence of the well known convergence in $L^{2}\left(\Lambda, L^{2}\left(\mathbb{R}_{y}^{p}\right)\right)$.

Thus, for $\varepsilon$ sufficiently small, $\Pi(x)\left(M_{\varepsilon} \tilde{u}_{l}\right)(x, \cdot)$ span $\operatorname{Ran} \Pi(x)$ for $x \in \bigcup_{j} \Omega_{j}$, and by Gram-Schmidt we obtain from these functions an orthonormal basis $u_{l}(x)$ of Ran $\Pi(x)$. This basis clearly satisfies (1.8), and (1.9) follows from (1.4) since $U_{j}(x)\left(M_{\varepsilon} \tilde{u}_{l}\right)(x, \cdot) \in C^{\infty}\left(\Omega_{j}, H^{2}\left(\mathbb{R}_{y}^{p}\right)\right)$. To modify the $u_{l}(x)$ outside $\lambda_{1}^{-1}(I)$ we fix a smooth partition of unity $\zeta, \zeta_{0}$ subordinate to $\Omega, \mathbb{R}^{n} \backslash \lambda_{1}^{-1}(I)$. Since $u_{l}(x)$ is continuous on the compact set $\bar{\Omega}$, we find for each $\varepsilon>0$ a cover $\left\{B_{j}\right\}_{j=1}^{N}$ of $\bar{\Omega}$ by small open sets, points $x_{j} \in B_{j}$, and a partition of unity $\chi_{j}$ subordinate to $B_{j}$, such 
that

$$
\left\|u_{l}(x)-\sum_{j=1}^{N} \chi_{j}(x) u_{l}\left(x_{j}\right)\right\|_{L^{2}\left(\mathbb{R}_{y}^{p}\right)} \leqq \varepsilon, \quad(x \in \Omega) .
$$

Choosing $M$ orthonormal functions $g_{l} \in H^{2}\left(\mathbb{R}_{y}^{p}\right)$ in the orthogonal complement of $\operatorname{Span}\left\{u_{l}\left(x_{j}\right) ; 1 \leqq l \leqq M, 1 \leqq j \leqq N\right\}$ we set

$$
\tilde{w}_{l}(x)=\zeta(x) u_{l}(x)+\zeta_{0}(x) g_{l} .
$$

Then it follows from (1.12) that

$$
\left\langle\tilde{w}_{l}(x), \tilde{w}_{k}(x)\right\rangle_{y}=\delta_{l k}+\mathcal{O}(\varepsilon),
$$

and orthonormalizing these functions (for $\varepsilon$ sufficiently small) yields

$$
\left(w_{1}(x), \ldots, w_{M}(x)\right)=\left(\tilde{w}_{1}(x), \ldots, \tilde{w}_{M}(x)\right) F^{-1 / 2}(x), \quad F(x)=\left(\left\langle\tilde{w}_{l}(x), \tilde{w}_{k}(x)\right\rangle_{y}\right)
$$

which verifies all the assertions of the lemma.

Next we fix $\zeta \in C_{0}^{\infty}(\Omega)$ with $0 \leqq \zeta \leqq 1$ and $\zeta=1$ in a neighbourhood $\mathscr{M}$ of $\lambda_{1}^{-1}(I)$ as in the proof of Lemma 1.1 and set

$$
Q^{\zeta}(x)=-\Delta_{y}+\zeta(x)\{V(x, y)+W(x)\} .
$$

Then $Q^{\zeta}(x)=Q(x)$ on $\lambda_{1}^{-1}(I=(-\infty, b])$ and

$$
Q^{\zeta}(x)>b, \quad\left(x \in \mathbb{R}^{n} \backslash \lambda_{1}^{-1}(I)\right) .
$$

In fact, for $\varphi \in H^{2}\left(\mathbb{R}^{p}\right)$ with $\|\varphi\|=1$ we have

$$
\left\langle Q^{\zeta}(x) \varphi, \varphi\right\rangle \geqq \zeta(x)\langle Q(x) \varphi, \varphi\rangle \geqq \min \{\langle Q(x) \varphi, \varphi\rangle, 0\} \geqq \min \left\{\lambda_{1}(x), 0\right\},
$$

proving (1.15). In particular, using (H3) and Lemma 1.1 this ensures that

$$
\hat{\Pi}(x) Q^{\zeta}(x) \hat{\Pi}(x)-\lambda>0, \quad\left(\lambda \in I, x \in \mathbb{R}^{n}\right),
$$

where $\hat{\Pi}(x)=1-\Pi(x)$ and $\Pi(x)$ denotes the orthogonal projection on Span $\left\{w_{1}(x), \ldots, w_{M}(x)\right\}$. This will be crucial for inverting the following Grushin operator associated with $P^{\zeta}=-h^{2} \Delta_{y}+Q^{\zeta}(x)+h^{2} p\left(\partial_{y}\right)$ :

$$
\mathscr{P}_{\lambda}=\left[\begin{array}{cccc}
P^{\zeta}-\lambda & w_{1} & \ldots & w_{M} \\
\left\langle\cdot, w_{1}\right\rangle_{y} & 0 & \ldots & 0 \\
\vdots & \vdots & & \vdots \\
\left\langle\cdot, w_{M}\right\rangle_{y} & 0 & \ldots & 0
\end{array}\right]
$$

which is selfadjoint as an operator in $L^{2}\left(\mathbb{R}^{n+p}\right) \oplus \bigoplus_{j=1}^{M} L^{2}\left(\mathbb{R}^{n}\right) \simeq L^{2}\left(\mathbb{R}^{n}\right.$; $\left.L^{2}\left(\mathbb{R}^{p}\right) \oplus \mathbb{C}^{M}\right)=: \mathscr{H}$. Here $w_{j}$ denotes multiplication by $w_{j}$ as an operator from $L^{2}\left(\mathbb{R}^{n}\right)$ to $L^{2}\left(\mathbb{R}^{n+p}\right)$ and $\left\langle\cdot, w_{j}\right\rangle_{y}$ its adjoint.

Before stating the main result of this section, let us recall the following notational convention: A family of bounded operators $A(h)$ from $H^{k}\left(\mathbb{R}^{n}, H_{1}\right)$ to $L^{2}\left(\mathbb{R}^{n}, H_{2}\right)$, where $H_{1}, H_{2}$ are Hilbert spaces and $h \in\left(0, h_{0}\right]$ with $h_{0}$ sufficiently small, is called $h$-admissible of order $k$, if for $N$ sufficiently large

$$
A(h)=\sum_{j=0}^{N} h^{j} \mathrm{Op}\left(a_{j}(x, \xi)\right)+h^{N} R_{N}(h),
$$


where $R_{N}$ is uniformly bounded from $L^{2}\left(\mathbb{R}^{n} ; H_{1}\right)$ to $L^{2}\left(\mathbb{R}^{n} ; H_{2}\right)$ for $0<h \leqq h_{0}$, $a_{j} \in C^{\infty}\left(T^{*} \mathbb{R}^{n} ; \mathscr{L}\left(H_{1}, H_{2}\right)\right)$ with $\left\|\partial^{\alpha} a_{j}(x, \xi)\right\|_{\mathscr{L}\left(H_{1}, H_{2}\right)} \leqq C_{\alpha}\langle\xi\rangle^{k}$ for all $\alpha \in \mathbb{N}^{2 n}$ uniformly in $(x, \xi) \in T^{*} \mathbb{R}^{n}$, and $\operatorname{Op}\left(a_{j}(x, \xi)\right)$ denotes the pseudodifferential operator which for $\varphi \in C_{0}^{\infty}\left(\mathbb{R}^{n}, H_{1}\right)$ is defined by the oscillatory integral

$$
\mathrm{Op}\left(a_{j}(x, \xi)\right) \varphi=(2 \pi h)^{-n / 2} \int e^{i\left(x-x^{\prime}\right) \xi / h} a_{j}(x, \xi) \varphi\left(x^{\prime}\right) d x^{\prime} d \xi .
$$

A slight generalization of the Calderon-Vaillancourt theorem then yields that $\mathrm{Op}\left(a_{j}\right)$ extends uniquely to a bounded map from $H^{k}\left(\mathbb{R}^{n}, H_{1}\right)$ to $L^{2}\left(\mathbb{R}^{n}, H_{2}\right)$ (see e.g. [GMS, BK]).

We shall call the formal series

$$
\sum_{j=0}^{\infty} h^{j} a_{j}(x, \xi)=a(x, \xi, h),
$$

which is uniquely determined by the $h$-admissible operator, the symbol $\sigma(A(h))$ of $A(h)$. Composition of $h$-admissible operators $A(h), B(h)$ induces the product of symbols

$$
(a \# b)(x, \xi, h)=\sigma(A(h) \circ B(h))
$$

which by the usual symbolic calculus is given by [BK, GMS]

$$
(a \# b)(x, \xi, h)=\sum_{\alpha \in \mathbb{N}^{n}} \frac{h^{|\alpha|}}{i^{|\alpha|} \alpha !} \partial_{\xi}^{\alpha} a(x, \xi, h) \partial_{x}^{\alpha} b(x, \xi, h),
$$

assuming of course that the image of $B$ matches the domain of $A$.

We are now ready to state the main result of this section as

Theorem 1.2. Assume $\mathrm{H} 1-\mathrm{H} 4$. Let $\Omega_{0} \subset \mathbb{R}^{n}$ be open and disjoint from $\Omega$ such that $\left\{\Omega_{j}\right\}_{j=0}^{r}$ cover $\mathbb{R}^{n}$. Then the Grushin operator $\mathscr{P}_{\lambda}$ is invertible for all $\lambda \in I$, and $\bmod \mathcal{O}\left(h^{\infty}\right)$ its inverse is given by

$$
\mathscr{E}=\mathscr{P}_{\lambda}^{-1}=\sum_{j=0}^{r} \mathscr{U}_{j}^{-1} \chi_{j} \mathscr{E}_{j} \mathscr{U}_{j} \chi_{j}
$$

where $\mathscr{E}_{j}$ is an h-admissible operator which is bounded from $L^{2}\left(\mathbb{R}^{n+p}\right) \oplus \bigoplus_{k=1}^{M} H^{2}\left(\mathbb{R}^{n}\right)$ to $H^{2}\left(\mathbb{R}^{n+p}\right) \oplus \bigoplus_{k=1}^{M} L^{2}\left(\mathbb{R}^{n}\right), \chi_{j} \in C^{\infty}\left(\Omega_{j}\right)$ with $\operatorname{supp} \chi_{j} \subset \Omega_{j}$ and

$$
\mathscr{U}_{j}=\left[\begin{array}{cc}
U_{j} & 0 \\
0 & 1
\end{array}\right]
$$

where $U_{j}$ acts on $L^{2}\left(\mathbb{R}_{x}^{n} \times \mathbb{R}_{y}^{p}\right)$ according to (1.5) and $U_{0}=\mathbf{1}$.

Furthermore, writing $\mathscr{E}=\left[\begin{array}{cc}E(\lambda) & E_{+}(\lambda) \\ E_{-}(\lambda) & E_{-+}(\lambda)\end{array}\right], E_{-+}(\lambda)$ is h-admissible, its principal symbol is the $M \times M$ matrix

$$
B(x, \xi ; \lambda)=\lambda \mathbf{1}-\left(\left\langle w_{l}(x),\left(\xi^{2}+Q^{\zeta}(x)\right) w_{k}(x)\right\rangle_{y}\right), \quad 1 \leqq k, l \leqq M,
$$

and we have $\forall \lambda \in I$ :

$$
\lambda \in S p\left(P^{\zeta}\right) \Leftrightarrow 0 \in \operatorname{Sp}\left(E_{-+}(\lambda)\right) .
$$

Remark 1.2. i) Without loss of generality we may assume that the set $\mathscr{M}$ on which $\zeta=1$ satisfies hypothesis (H3) with $\Omega$ replaced by $\mathscr{M}$. One may then use the 
arguments of [HS1] to show that both $S p(P)$ and $S p\left(P^{\zeta}\right)$ coincide in $I=(-\infty, b]$ with the spectrum $S p\left(P_{\mathscr{M}}\right)$ of the Dirichlet realization of $P$ on $\mathscr{M}$ up to $\mathcal{O}\left(e^{-\delta / h}\right)$ as $h \downarrow 0$, where $0<\delta<d\left(\lambda_{1}^{-1}(I), \partial \mathscr{M}\right)$ and $d$ denotes the distance in the Agmon metric $d s^{2}=\max \left\{\lambda_{1}(x)-b, 0\right\} d x^{2}$.

This follows by combining a rough estimate on the number of eigenvalues of $P$ in $I$ with the Agmon type estimate

$$
\left\|\nabla\left(e^{(1-\varepsilon) d / h} \varphi\right)\right\|+\left\|e^{(1-\varepsilon) d / h} \varphi\right\|=\mathcal{O}(1) \quad \text { as } \quad h \downarrow 0(\varepsilon>0),
$$

where $d(x)=d\left(x, \lambda_{1}^{-1}(I)\right)$ and $\varphi$ is any eigenfunction of $P_{\mathscr{M}}$ (or $P$ or $P^{\zeta}$ in the respective domain) with eigenvalue in $I$.

In order to study $S p(P) \cap I$ it thus suffices to study $P^{\zeta}$, which has the advantage of leading to the Grushin problem (1.17) depending - at least after local coordinate transformations - smoothly on $x$. If, for instance, $Q(x)$ depends smoothly on $x$ globally in $\mathbb{R}^{n}$ [or does so after a finite number of coordinate changes as in (H2)], then Theorem 1.2 remains true for the initial operator $P$ replacing the smoothed out operator $P^{\zeta}$ in (1.17).

ii) One could also have worked with $f(P)$ instead of $P^{\zeta}$, where $f \in C^{\infty}(\mathbb{R}), f(\lambda)=\lambda$ on $(-\infty, b], f=$ const on $\left[b^{\prime}, \infty\right)$ for some $b^{\prime}>b$. Then the operator $E_{-+}(\lambda)$ in $(1.21)$ would have been replaced by a bounded operator $\widetilde{E}_{-+}(\lambda)$ which also is $h$-admissible, but less pleasant to work with than $E_{-+}(\lambda)$. In particular, it does not via its symbol connect in any obvious way with the WKB constructions of Sect. 3.

iii) It follows from (1.20) that

$$
B(x, \xi ; \lambda)=\lambda-\xi^{2} 1-A(x),
$$

where $A(x)$ is a symmetric matrix satisfying $A(x)>b \mathbf{1}$ for $x \in \mathbb{R}^{n} \backslash \lambda_{1}^{-1}(I)$.

Proof. The idea of the proof is in localizing $\mathscr{P}_{\lambda}$ by means of a partition of unity to the coordinate patches $\Omega_{j}$, where conjugation by $\mathscr{U}_{j}$ yields a pseudodifferential operator with a smooth operator valued symbol. Inverting its principal symbol defines an approximate inverse which then can be corrected to yield $\mathscr{P}_{\lambda}^{-1}$ in the form (1.19). We choose $\chi_{j} \in C_{0}^{\infty}\left(\Omega_{j}\right)$ for $j \geqq 1, \chi_{0} \in C^{\infty}\left(\Omega_{0}\right)$ with $\sum_{j=0}^{r} \chi_{j}^{4}=1$ and set

$$
\begin{aligned}
P_{j} & =U_{j} \chi_{j} P^{\zeta} U_{j}^{-1} \chi_{j} \\
& =h^{2} \chi_{j}\left(D_{x}+J(x, y) D_{y}+K(x, y)\right)^{2} \chi_{j}+h^{2} \chi_{j} p\left({ }_{j} \nabla_{y}\right) \chi_{j}+\chi_{j} Q_{j}^{\zeta}(x) \chi_{j},
\end{aligned}
$$

where

$$
\begin{aligned}
J(x, y) & =\left(\partial_{x} G_{j}\right)^{T}\left(x, y^{\prime}=F_{j}(x, y)\right), \\
K(x, y) & =\left|\operatorname{det} \partial_{y} F_{j}\right|^{1 / 2} D_{x}\left|\operatorname{det} \partial_{y^{\prime}} G_{j}\left(x, y^{\prime}=F_{j}(x, y)\right)\right|^{1 / 2}, \\
{ }_{j} \nabla_{y} & =\nabla_{y}+\left|\operatorname{det} \partial_{y} F_{j}\right|^{1 / 2} \nabla_{y}\left|\operatorname{det} \partial_{y^{\prime}} G_{j}\left(x, y^{\prime}=F_{j}(x, y)\right)\right|^{1 / 2}, \\
Q_{j}^{\zeta}(x) & =U_{j}(x) Q^{\zeta}(x) U_{j}^{-1}(x)
\end{aligned}
$$

and $G_{j}(x, \cdot)$ is the inverse diffeomorphism of $F_{j}(x, \cdot)$. Clearly $P_{j}$ is selfadjoint in $L^{2}\left(\mathbb{R}^{n+p}\right)$ with domain containing $\mathscr{D}(P)$ and semibounded from below uniformly in $h$; in fact, we have $P^{\zeta} \geqq-\gamma$ for some $\gamma>0$, and thus

$$
\left\langle P_{j} u, u\right\rangle=\left\langle P^{\zeta} U_{j}^{-1} \chi_{j} u, U_{j}^{-1} \chi_{j} u\right\rangle \geqq-\gamma\left\|\chi_{j} u\right\|^{2} \geqq-\gamma\|u\|^{2} .
$$


We shall now consider the localized Grushin operator

$$
\mathscr{P}_{j}=\mathscr{U}_{j} \chi_{j} \mathscr{P}_{\lambda} \mathscr{U}_{j}^{-1} \chi_{j}=\left[\begin{array}{cccc}
P_{j}-\lambda \chi_{j}^{2} & \chi_{j}^{2} w_{1, j} & \ldots & \chi_{j}^{2} w_{M, j} \\
\chi_{j}^{2}\left\langle\cdot, w_{1, j}\right\rangle_{y} & & & \\
\vdots & & 0 & \\
\chi_{j}^{2}\left\langle\cdot, w_{M, j}\right\rangle_{y} & &
\end{array}\right],
$$

where $w_{l, j}=U_{j} w_{l}$.

In view of $(\mathrm{H} 2)$ and Lemma $1.1 \mathscr{P}_{j}$ is a pseudodifferential operator with an $\quad \mathscr{L}\left(H^{2}\left(\mathbb{R}^{p}\right) \oplus \mathbb{C}^{M} ; \quad L^{2}\left(\mathbb{R}^{p}\right) \oplus \mathbb{C}^{M}\right)$-valued symbol, bounded from $H^{2}\left(\mathbb{R}^{n+p}\right) \oplus \bigoplus_{k=1}^{M} L^{2}\left(\mathbb{R}^{n}\right)$ to $L^{2}\left(\mathbb{R}^{n+p}\right) \oplus \bigoplus_{k=1}^{M} H^{2}\left(\mathbb{R}^{n}\right)$, and by (1.22) its $h$-principal symbol is given by

$$
p_{j}(x, \xi)=\chi_{j}^{2}(x)\left[\begin{array}{cccc}
\xi^{2}+Q_{j}^{\zeta}(x)-\lambda & w_{1, j}(x) & \ldots & w_{M, j}(x) \\
\left\langle\cdot, w_{1, j}(x)\right\rangle_{y} & & & \\
\vdots & & 0 & \\
\left\langle\cdot, w_{M, j}(x)\right\rangle_{y} & & &
\end{array}\right]=\chi_{j}^{2}(x) \tilde{p}_{j}(x, \xi) .
$$

Using (1.16) one readily verifies that for all $\lambda \in I,(x, \xi) \in T^{*} \Omega_{j}, \tilde{p}_{j}(x, \xi)$ is invertible with inverse

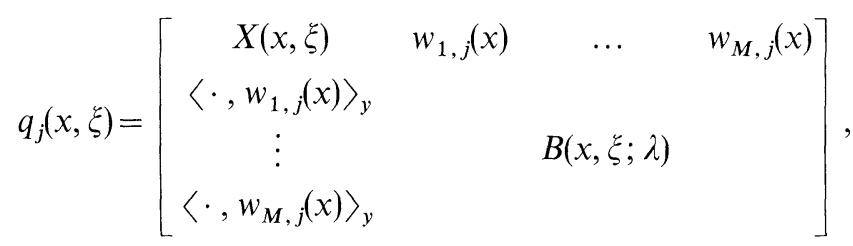

$$
\begin{aligned}
& X(x, \xi)=\hat{\Pi}_{j}(x)\left(\hat{\Pi}_{j}(x)\left(\xi^{2}+Q_{j}^{\zeta}(x)\right) \hat{\Pi}_{j}(x)-\lambda\right)^{-1} \hat{\Pi}_{j}(x),
\end{aligned}
$$

where the $M \times M$ matrix $B(x, \xi ; \lambda)$ is given by $(1.20)$ - independently of $j !-$ and $\hat{\Pi}_{j}(x)=U_{j}(x) \hat{\Pi}(x) U_{j}^{-1}(x)$ denotes the projection on the orthogonal complement of $\operatorname{Span}\left\{w_{1, j}(x), \ldots, w_{M, j}(x)\right\}$.

Setting

$$
Q_{j}=\chi_{j} O p\left(q_{j}(x, \xi)\right) \chi_{j}, \quad Q=\sum_{j=0}^{r} \mathscr{U}_{j}^{-1} \chi_{j}^{2} Q_{j} \mathscr{U}_{j}
$$

one has

$$
\mathscr{P}_{\lambda} Q=\sum_{j=0}^{r}\left\{\mathscr{U}_{j}^{-1} \mathscr{P}_{j} Q_{j} \mathscr{U}_{j}+\left[\mathscr{P}_{\lambda}, \chi_{j}\right] \mathscr{U}_{j}^{-1} \chi_{j} Q_{j} \mathscr{U}_{j}\right\}
$$

Note that $Q_{j}$ is bounded as a map from $\mathscr{D}\left(Q_{j}\right):=L^{2}\left(\mathbb{R}^{n+p}\right) \oplus \bigoplus_{k=1}^{M} H^{2}\left(\mathbb{R}^{n}\right)$ to $H^{2}\left(\mathbb{R}^{n+p}\right) \oplus \bigoplus_{k=1}^{M} L^{2}\left(\mathbb{R}^{n}\right)=\mathscr{D}\left(\mathscr{P}_{\lambda}\right)$ as follows by inspection of each matrix element of $Q_{j}$. Thus the composition $\mathscr{P}_{j} Q_{j}$ is well defined as a bounded operator in $\mathscr{D}\left(Q_{j}\right)$ (vice versa $Q_{j} \mathscr{P}_{j}$ is bounded on $\left.\mathscr{D}\left(\mathscr{P}_{\lambda}\right)\right)$, and we shall now apply the pseudodifferential calculus for operator valued symbols separately to each matrix element of $\mathscr{P}_{j} Q_{j}$ to 
get that

$$
\mathscr{P}_{j} Q_{j}=\chi_{j}^{4}+h \chi_{j, 1} R_{j},
$$

where $\chi_{j, 1} \in C^{\infty}\left(\Omega_{j}\right)$ with $\chi_{j, 1}=1$ on $\operatorname{supp} \chi_{j}$, supp $\chi_{j, 1} \subset \Omega_{j}$, and $R_{j}$ is an $h$-admissible operator which on $\mathscr{D}\left(Q_{j}\right)$ is uniformly bounded in $h$. In fact, with $X(x, \xi)$ as in (1.26) we have for $m=0,1,2$

$$
\left\|\langle\xi\rangle^{m} \partial_{x, \xi}^{\alpha} X(x, \xi)\right\|_{\mathscr{L}\left(L^{2}\left(\mathbb{R}_{y}^{p}\right), H^{2-m}\left(\mathbb{R}_{y}^{p}\right)\right)} \leqq C_{\alpha},
$$

while a short calculation gives the principal symbol of $R_{j}$ as

$$
\begin{aligned}
\sigma_{\mathrm{pr}}\left(R_{j}\right)= & \frac{2}{i} \sum_{i=1}^{n}\left\{\chi_{j}^{4}(x)\left[\begin{array}{cccc}
\xi_{i} \partial_{x_{i}} X(x, \xi) & \xi_{i} \partial_{x_{i}} w_{1, j} & \ldots & \xi_{i} \partial_{x_{i}} w_{M, j} \\
0 & 0
\end{array}\right]\right. \\
& \left.+4 \chi_{j}^{3}(x) \partial_{x_{i}} \chi_{j}(x)\left[\begin{array}{cccc}
\xi_{i} X(x, \xi) & \xi_{i} w_{1, j} & \ldots & \xi_{i} w_{M, j} \\
0 & & 0
\end{array}\right]\right\} .
\end{aligned}
$$

Thus $R_{j, 0}:=\operatorname{Op}\left(\sigma_{\mathrm{pr}}\left(R_{j}\right)\right)$ is even bounded from $L^{2}\left(\mathbb{R}^{n+p}\right) \oplus \underset{k=1}{M} H^{1}\left(\mathbb{R}^{n}\right)$ to $\mathscr{D}\left(Q_{j}\right)$, and one readily checks using the symbolic calculus that

$$
R_{j}=R_{j, 0}+h R_{j, 1}(h),
$$

where the pseudodifferential operator $R_{j, 1}(h)$ is uniformly bounded from $L^{2}\left(\mathbb{R}^{n} ; L^{2}\left(\mathbb{R}^{p}\right) \oplus \mathbb{C}^{M}\right) \cong L^{2}\left(\mathbb{R}^{n+p}\right) \oplus \underset{k=1}{M} L^{2}\left(\mathbb{R}^{n}\right)$ to $\mathscr{D}\left(Q_{j}\right)$. This proves (1.29).

Since furthermore the pseudodifferential operator

$$
\left[\begin{array}{cc}
h D_{x} & 0 \\
0 & 0
\end{array}\right] \chi_{j} Q_{j}
$$

is of the same type as the principal part $R_{j, 0}$ of $R_{j}$, the operator $\mathscr{U}_{j}\left[\mathscr{P}_{\lambda}, \chi_{j}\right] \mathscr{U}_{j}^{-1} \chi_{j} Q_{j}$ - corresponding to the second member on the right-hand side of $(1.28)$ - also is of the form $h \tilde{\chi}_{j, 1} \widetilde{R}_{j}$, where $\tilde{\chi}_{j, 1}$ and $\widetilde{R}_{j}$ have the same properties as $\chi_{j, 1}$ and $R_{j}$.

Redefining $\chi_{j, 1}$ and $R_{j}$ we thus have

$$
\mathscr{P}_{\lambda} Q=\mathbf{1}+h \mathscr{R}, \quad \text { where } \quad \mathscr{R}=\sum_{j=0}^{r} \mathscr{U}_{j}^{-1} \chi_{j, 1} R_{j} \mathscr{U}_{j} \chi_{j, 1} .
$$

In order to compute $\mathscr{P}_{\lambda}^{-1}$ from a geometric series, we essentially have to compute a change of local coordinates in the overlap regions $\Omega_{j} \cap \Omega_{1}$ and use the fact that the $h$-pseudodifferential operators $R_{j}$ are still pseudolocal in the base space $\mathbb{R}_{x}^{n}$. We need

Lemma 1.3. For all $k \in \mathbb{N}$ one has $\bmod \mathcal{O}\left(h^{\infty}\right)$

$$
\mathscr{R}^{k}=\sum_{j=0}^{r} \mathscr{U}_{j}^{-1} \chi_{j, k} A_{j, k} \mathscr{U}_{j} \chi_{j, k},
$$

where $\chi_{j, k} \in C^{\infty}\left(\Omega_{j}\right)$ equals 1 on $\operatorname{supp} \chi_{j, k-1}\left(\operatorname{setting} \chi_{j}=\chi_{j, 0}\right), \bigcup_{k \in \mathbb{N}} \operatorname{supp} \chi_{j, k} \subset C \Omega_{j}$ and $A_{j, k}$ is an h-admissible operator which is uniformly bounded in $h$ on $\mathscr{D}(Q)=L^{2}\left(\mathbb{R}^{n+p}\right) \oplus \bigoplus_{k=1}^{M} H^{2}\left(\mathbb{R}^{n}\right)$.

Proof of Lemma 1.3. It follows readily from (1.25) that

$$
\mathscr{U}_{j}(x) \mathscr{U}_{l}^{-1}(x) q_{l}(x, \xi) \mathscr{U}_{l}(x) \mathscr{U}_{j}^{-1}(x)=q_{j}(x, \xi) \quad\left(x \in \Omega_{j} \cap \Omega_{l}, 0 \leqq j, l \leqq r\right)
$$


and similarly for $\tilde{p}_{j}(x, \xi)$. Thus, using the symbolic calculus, one easily verifies that

$$
R_{j, l}^{(k+1)}:=\mathscr{U}_{j} \chi_{j, k} \mathscr{U}_{l}^{-1} \chi_{l, 1} R_{l} \mathscr{U}_{l} \chi_{l, 1} \mathscr{U}_{j}^{-1} \chi_{j, k+1} \quad(0 \leqq j, l \leqq r, k \in \mathbb{N})
$$

is $h$-admissible and uniformly bounded in $h$. This gives

$$
\mathscr{R}^{2}=\sum_{j, l=0}^{r}\left\{\mathscr{U}_{j}^{-1} \chi_{j, 1} R_{j} R_{j, l}^{(2)} \mathscr{U}_{j} \chi_{j, 2}+\mathscr{U}_{j}^{-1} \chi_{j, 1} R_{j} \mathscr{U}_{j} \mathscr{U}_{l}^{-1} \chi_{j, 1} \chi_{l, 1} R_{l}\left(1-\chi_{j, 2}^{2}\right) \mathscr{U}_{l} \chi_{l, 1},\right.
$$

where the last term is $\mathcal{O}\left(h^{\infty}\right)$ since $\operatorname{supp} \chi_{j, 1}$ is disjoint from $\operatorname{supp}\left(1-\chi_{j, 2}^{2}\right)$.

We then obtain (1.33) by induction on $k$ with

$$
A_{j, k}=\sum_{l_{2}, \ldots, l_{k}=0}^{r} R_{j} R_{j, l_{2}}^{(2)} \ldots R_{j, l_{k}}^{(k)} .
$$

To complete the proof of Theorem 1.2, we derive from (1.32),

$$
\begin{aligned}
\mathscr{P}_{\lambda}^{-1} & =Q\left\{1+\sum_{k=1}^{N}(-h)^{k} \mathscr{R}^{k}\right\}+\mathcal{O}\left(h^{N}\right) \\
& =Q\left\{1+\sum_{j=0}^{r} \mathscr{U}_{j}^{-1} \tilde{\chi}_{j}\left(\sum_{k=1}^{N}(-h)^{k} \chi_{j, k} A_{j, k} \chi_{j, k}\right) \mathscr{U}_{j} \tilde{\chi}_{j}\right\}+\mathcal{O}\left(h^{N}\right)
\end{aligned}
$$

where $\tilde{\chi}_{j} \in C^{\infty}\left(\Omega_{j}\right)$ with $\operatorname{supp} \tilde{\chi}_{j} \subset \Omega_{j}$ equals 1 on $\bigcup_{k \in \mathbb{N}} \operatorname{supp} \chi_{j, k}$.

Inserting (1.27) on the right-hand side we have represented $\mathscr{P}_{\lambda}^{-1}$ in the form (1.19), since $\chi_{l} A_{j, k}\left(1-\tilde{\chi}_{l}\right)=\mathcal{O}\left(h^{\infty}\right)$ and

$$
\mathscr{U}_{l} \chi_{l} \mathscr{U}_{j}^{-1} \tilde{\chi}_{j} A_{j, k} \mathscr{U}_{j} \tilde{\chi}_{j} \mathscr{U}_{l}^{-1} \tilde{\chi}_{l}
$$

is $h$-admissible in view of (1.37) and (1.35). By construction, one has a natural bijection between $\operatorname{Ker}(P-\lambda)$ and $\operatorname{Ker} E_{-+}(\lambda)$, which completes the proof of Theorem 1.2.

In order to relate Theorem 1.2 to the WKB expansions of Sect. 3 , it is convenient to state separately a few consequences of Theorem 1.2 and its proof. They essentially regard the behavior of $\mathscr{E}=\mathscr{P}_{\lambda}^{-1}$ under coordinate changes in the overlap region $\Omega_{j} \cap \Omega_{l}$.

Corollary 1.4. For each $\chi_{j} \in C_{0}^{\infty}\left(\Omega_{j}\right)$,

$$
\widetilde{\mathscr{E}}_{j}=\mathscr{U}_{j} \chi_{j} \mathscr{E} \mathscr{\mathscr { C }} \mathscr{U}_{j}^{-1} \chi_{j}
$$

is an h-admissible operator. Now fix $x_{0} \in \mathbb{R}^{n}$. Letting $I_{x_{0}}=\left\{j ; x_{0} \in \Omega_{j}\right\}$, we choose for $j \in I_{x_{0}}$ cut-off functions $\chi, \chi_{j} \in C_{0}^{\infty}\left(\Omega_{j}\right)$ with $\chi, \chi_{j}=1$ near $x_{0}$ and $\chi$ supported in the interior of $\left\{\chi_{j}=1\right\}$. Then

$$
\widetilde{\mathscr{E}}_{j} \mathscr{P}_{j} \chi=\chi+\mathcal{O}\left(h^{\infty}\right)=\mathscr{P}_{j} \widetilde{\mathscr{E}}_{j} \chi+\mathcal{O}\left(h^{\infty}\right),
$$

where $\mathscr{P}_{j}$ denotes the pseudodifferential operator $\mathscr{U}_{j} \chi_{j} \mathscr{P}_{\lambda}^{-1} \mathscr{U}_{j}^{-1} \chi_{j}$. In particular, we have

$$
\left(\sigma\left(\widetilde{\mathscr{E}}_{j}\right) \# \sigma\left(\mathscr{P}_{j}\right)\right)(x, \xi, h)=\mathbf{1}_{H^{2}\left(\mathbb{R}^{p}\right) \oplus \mathbb{C}^{m}}
$$

for all $j \in I_{x_{0}}$ and $x$ sufficiently close to $x_{0}$. 
Proof. Since

$$
\widetilde{E}_{j}=\sum_{l=0}^{r} \mathscr{U}_{j} \chi_{j} \mathscr{U}_{l}^{-1} \chi_{l} \mathscr{E}_{l} \mathscr{U}_{l} \chi_{l} \mathscr{U}_{j}^{-1} \chi_{j}+\mathcal{O}\left(h^{\infty}\right)
$$

one has to check that each individual term on the right-hand side of (1.41) is $h$-admissible. This follows combining the representation of $\mathscr{E}_{j}$ given by $(1.38)$ with (1.37) and the crucial result (1.35) on conjugation of all remainder terms $R_{j, l}$ by $\mathscr{U}_{l} \mathscr{U}_{j}^{-1}$. Furthermore

$$
\mathscr{P}_{j} \widetilde{\mathscr{E}}_{j} \chi=\mathscr{U}_{j} \chi_{j} \mathscr{P}_{\lambda} \mathscr{U}_{j}^{-1} \chi_{j} \mathscr{U}_{j} \chi_{j} \mathscr{E} \mathscr{U}_{j}^{-1} \chi_{j} \chi=\mathscr{U}_{j} \chi_{j} \mathscr{U}_{j}^{-1} \chi_{j} \chi+\mathscr{U}_{j} \chi_{j} \mathscr{P}_{\lambda}\left(1-\chi_{j}^{2}\right) \mathscr{E} \mathscr{U}_{j}^{-1} \chi_{j} \chi
$$

and the same for $\widetilde{\mathscr{E}} \mathscr{P}_{j} \chi$, which proves (1.39) in view of the disjoint support of $\left(1-\chi_{j}^{2}\right)$ and $\chi$.

Finally (1.40) follows from (1.39), since $\sigma\left(\widetilde{\mathscr{E}}_{j} \mathscr{P}_{j} \chi\right)(x, \xi, h)=\sigma\left(\widetilde{\mathscr{E}}_{j} \mathscr{P}_{j}\right)(x, \xi, h)$ for $x$ close to $x_{0}$, if $\chi=1$ near $x_{0}$.

As the first application of Theorem 1.2, we shall prove that the eigenvalues of $P$ can be approximated by those of the effective Hamiltonian.

Proposition 1.5. Assume that the conditions of Theorem 1.2 are satisfied with $M=1$. Let $P_{0}(h)$ denote the Dirichlet realization of

$$
-h^{2} \Delta_{x}+\lambda_{1}(x)+h^{2}\left\langle\left(-\Delta_{x}+p\left(\partial_{y}\right)\right) u_{1}(x, \cdot), u_{1}(x, \cdot)\right\rangle_{y}
$$

over $\Omega$. Put $I=(-\infty, a(h)]$, where $a(h)>\lambda_{0}$ is a function of $h$ such that

$$
a(h) \leqq \lambda_{0}+c h \quad \text { and } \quad \operatorname{dist}\left(a(h), S p\left(P_{0}(h)\right)\right) \geqq c^{-1} h^{2}, \quad \text { for some } c>0 .
$$

Then for $h>0$ small enough there exists a bijection $b: S p(P) \cap I \rightarrow S p\left(P_{0}\right) \cap I$ such that

$$
|b(\lambda)-\lambda|=\mathcal{O}\left(h^{5 / 2}\right), \quad \forall \lambda \in S p(P(h)) \cap I .
$$

Proof. According to the Remark following Theorem 1.2, $\lambda \in S p(P) \cap I$ if there is $\mu=\lambda+O\left(e^{-\varepsilon_{0} / h}\right)$ such that $0 \in S p\left(E_{-+}(\mu)\right)$. In addition the symbol of $E_{-+}(\lambda)$ can be computed:

$$
\begin{aligned}
\sigma\left(E_{-+}(\lambda)\right)= & \lambda-\left\{\xi^{2}+\left\langle w_{1}(x, \cdot), Q^{\zeta}(x) w_{1}(x, \cdot)\right\rangle_{y}\right. \\
& \left.+h^{2}\left(\left\langle\left(-\Delta_{x}+p(\partial y)\right) w_{1}(x, \cdot), w_{1}(x, \cdot)\right\rangle_{y}+\sum_{i, j} \xi_{i} \xi_{j} c_{i j}(x, \xi ; \lambda)\right)+\mathcal{O}\left(h^{3}\right)\right\},
\end{aligned}
$$

where $c_{i j}(\cdot, \cdot ; \lambda)$ is holomorphic in $\lambda \in I$ and $\left|\partial_{x, \xi}^{\alpha} c_{i j}(x, \xi ; \lambda)\right| \leqq c_{\alpha}\langle\xi\rangle^{-2}$. Put $F(\lambda)$ $=\lambda-E_{-+}(\lambda)$ and

$$
\tilde{F}=-h^{2} \Delta_{x}+\left\langle Q^{\zeta}(x) w_{1}(x, \cdot), w_{1}(x, \cdot)\right\rangle_{y}+h^{2}\left\langle\left(-\Delta_{x}+p\left(\partial_{y}\right)\right) w_{1}(x, \cdot), w_{1}(x, \cdot)\right\rangle_{y} .
$$

Since $Q^{\zeta}(x)=Q(x), w_{1}(x, \cdot)=u_{1}(x, \cdot)$ for $x \in \Omega$, the eigenvalues of $\widetilde{F}$ in $I$ are equal to those of $P_{0}$ modulo an error of order $O\left(e^{-\varepsilon_{0} / h}\right)$ for some $\varepsilon_{0}>0$. In addition, we deduce from (1.44) that

$$
\widetilde{F}_{-} \leqq F(\lambda) \leqq \widetilde{F}_{+}, \quad \text { where } \quad \widetilde{F}_{ \pm}=\widetilde{F} \pm c h^{2}\left(-h^{2} \Delta+h\right) \text {, for some } c \gg 1 \text {. }
$$

Now if $u$ is a normalized eigenfunction of $\widetilde{F}$ with eigenvalue $E(h) \in I$, we have:

$$
\left\|\left(\tilde{F}_{ \pm}-E(h)\right) u\right\| \leqq c h^{3}+c h^{2}\left\|-h^{2} \Delta_{x} u\right\| \leqq c^{\prime} h^{3}+c^{\prime} h^{2}\left\|\left(\tilde{\lambda}_{1}(x)-\lambda_{0}\right) u\right\| .
$$


Here $\tilde{\lambda}_{1}(x)=\left\langle Q^{\zeta}(x) w_{1}(x), w_{1}(x)\right\rangle$. Let $\phi(x)$ denote the Agmon distance of $x$ to $\left\{\tilde{\lambda}_{1}=\lambda_{0}\right\}$ in the metric $\left(\tilde{\lambda}_{1}(x)-\lambda_{0}\right) d x^{2}$. In the domain $\tilde{\lambda}_{1}(x)-\lambda_{0} \geqq c h^{1 / 2}$, we can then easily estimate:

$$
\phi(x) \geqq \delta_{0} h^{3 / 4}, \quad \text { for some } \delta_{0}>0
$$

Consequently, by the exponential decay of the eigenfunctions,

$$
\begin{aligned}
\left\|\left(\tilde{\lambda}_{1}(x)-\lambda_{0}\right) u\right\| & \leqq c h^{1 / 2}+\left\{\tilde{\lambda}_{1} \geqq \lambda_{0}+c h^{1 / 2}\right. \\
& \leqq c^{\prime} h^{1 / 2}
\end{aligned}
$$

This proves:

$$
\left\|\left(\tilde{F}_{ \pm}-E(h)\right) u\right\| \leqq c h^{5 / 2} .
$$

Similarly if $v$ is a normalized eigenfunction of $\widetilde{F}_{+}$or $\widetilde{F}_{-}$with eigenvalue $\widetilde{E}(h) \in I$, we also have:

$$
\|(\widetilde{F}-\tilde{E}(h)) v\| \leqq c h^{5 / 2} .
$$

By the min-max principle and the above estimates, we deduce that for $h>0$ small enough, $F(\lambda)$ and $\widetilde{F}$ have the same number of eigenvalues in $I$ and there is a bijection $b_{\lambda}: S p(F(\lambda)) \cap I \rightarrow S p(\tilde{F}) \cap I$ such that

$$
\left|b_{\lambda}(\mu)-\mu\right|=\mathcal{O}\left(h^{5 / 2}\right) \text { for } \mu \in \operatorname{Sp}(F(\lambda)) \cap I .
$$

Let $\left\{v_{j}(\lambda)\right\}$ be the eigenvalues of $F\left(\lambda_{s}\right)$ in $I$ arranged in increasing order. As in [Ma2, Sect. 4], we have:

$$
\frac{\partial v_{j}}{\partial \lambda}(\lambda) \leqq 0, \quad \forall j
$$

and for each $j$, the equation $\lambda=v_{j}(\lambda)$ has exactly one solution in $I$. This ends the proof of Proposition 1.5.

\section{Application to Molecular Schrödinger Operators}

We shall now write $x=\left(x_{1}, \ldots, x_{m}\right) \in \mathbb{R}^{3 m}$ for the (Jacobi coordinates of) $(m+1)$ nuclei in the center of mass frame; $y=\left(y_{1}, \ldots, y_{q}\right) \in \mathbb{R}^{p}, p=3 q$, for the position of $q$ electrons, and consider a molecular potential of the form

$$
\begin{aligned}
V(x, y) & =\sum_{\substack{1 \leqq i \leqq p \\
1 \leqq j \leqq m}} V_{i j}\left(\left|A_{j} x-y_{i}\right|\right)+\sum_{1 \leqq i<j \leqq p} \tilde{V}_{i j}\left(\left|y_{i}-y_{j}\right|\right) \\
W(x) & =\sum_{1 \leqq i<j \leqq m+1} W_{i j}\left(\left|A_{i} x-A_{j} x\right|\right),
\end{aligned}
$$

where the constant $3 \times 3 m$ matrices $A_{j}$ of the form

$$
A_{j} x=\sum_{k=1}^{m} a_{j, k} x_{k}, \quad a_{j, k} \in \mathbb{R}
$$

are due to the removal of the center of mass motion, and we assume that the 2-body central potentials $V_{i j}(|s|), \widetilde{V}_{i j}(|s|), W_{i j}(|s|)$ are $\Delta_{s}$-compact in $\mathbb{R}^{3}$ and verify (writing $V$ for any of the $V_{i j}$ or $\widetilde{V}_{i j}$, and $W$ for any $W_{i j}$ ) 
(H mol) $V, W \in C^{\infty}\left(\mathbb{R}^{3} \backslash\{0\}\right), W \geqq 0$ and for all $k \in \mathbb{N}$ one has

$$
\sup _{r \geqq 1}\left|V^{(k)}(r)\right|+\sup _{r \leqq 1} \frac{r^{k}\left|V^{(k)}(r)\right|}{1+|V(r)|} \leqq C_{k}<\infty .
$$

Furthermore we assume that the "isotopic operator" $p\left(\partial_{y}\right)$ in (1.1) commutes with rotations in each $\mathbb{R}_{y_{j}}^{3}$ (this is the case in the physical situation, where it is generated by removal of the center of mass motion).

We remark that the above assumption of rotational invariance covers the case of physical potentials, but is not essential for our methods to work. We can obtain the same type of results as long as the potential wells are nondegenerate.

We denote by $\mathscr{C}=\left\{x \in \mathbb{R}^{3 m} ; A_{j} x=A_{i} x\right.$ for some $\left.i \neq j\right\}$ the set of points corresponding to collisions of the $m+1$ nuclei (which is a finite union of planes of codimension 3), and we shall show that in $\mathbb{R}^{3 m} \backslash \mathscr{C}$ there exist local coordinate transformations as in $(\mathrm{H} 2)$.

For $x_{0}=\left(x_{1}^{0}, \ldots, x_{m}^{0}\right) \in \mathbb{R}^{3 m} \backslash \mathscr{C}$ fixed, there exist $f_{j} \in C_{0}^{\infty}\left(\mathbb{R}^{3}, \mathbb{R}\right)$ with $f_{j}\left(A_{k} x_{0}\right)=\delta_{j k}$ $(1 \leqq j, k \leqq m+1)$, and we set

$$
F_{x_{0}}(x, s)=s+\sum_{j=1}^{m+1} A_{j}\left(x-x_{0}\right) f_{j}(s), \quad\left(s \in \mathbb{R}^{3}, x \in \mathbb{R}^{3 m}\right) .
$$

This is similar to the transformation introduced by Hunziker [Hu]. For $x$ in a sufficiently small neighborhood $\Omega_{x_{0}}$ of $x_{0}$ it verifies

$$
\begin{aligned}
& F_{x_{0}}(x, \cdot) \text { is a diffeomorphism of } \mathbb{R}^{3} \text { which depends analytically on } x, \\
& F_{x_{0}}(x, s)=s \text { for }|s| \text { sufficiently large and } \\
& F_{x_{0}}\left(x, A_{j} x_{0}\right)=A_{j} x, \quad(1 \leqq j \leqq m+1) .
\end{aligned}
$$

In particular, the transformation

$$
F_{0}(x, y):=\left(F_{x_{0}}\left(x, y_{1}\right), \ldots, F_{x_{0}}\left(x, y_{q}\right)\right), \quad\left(x \in \Omega_{x_{0}}, y \in \mathbb{R}^{3 q}\right)
$$

is in $C^{\infty}\left(\Omega_{x_{0}} \times \mathbb{R}^{p} ; \mathbb{R}^{p}\right)$ and satisfies the estimate

$$
\begin{gathered}
\forall \alpha \in \mathbb{N}^{3 m} \exists C_{\alpha}>0 \forall x \in \Omega_{x_{0}} \forall y, y^{\prime} \in \mathbb{R}^{p}: \\
\frac{1}{C_{0}}\left|y-y^{\prime}\right| \leqq\left|F_{0}(x, y)-F_{0}\left(x, y^{\prime}\right)\right| \leqq C_{0}\left|y-y^{\prime}\right|, \\
\left|\partial_{x}^{\alpha} F_{0}(x, y)-\partial_{x}^{\alpha} F_{0}\left(x, y^{\prime}\right)\right| \leqq C_{\alpha}\left|y-y^{\prime}\right|, \\
\left|\partial_{x}^{\alpha} F_{0}(x, y)\right| \leqq C_{0}, \quad(|\alpha| \geqq 1) .
\end{gathered}
$$

We shall use (2.6) to verify (1.2) in hypothesis $(\mathrm{H} 2)$ which is the essential point in applying the result of Sect. 1 to molecular Schrödinger operators of the form $(\mathrm{H} \mathrm{mol})$. Note that $V\left(x, F_{0}(x, y)\right)$ is a sum of terms of the form

a) $W_{i j}\left(\left|A_{i} x-A_{j} x\right|\right) \in C^{\infty}\left(\mathbb{R}^{3 n} \backslash \mathscr{C}\right)$,

b) $\widetilde{V}_{i j}\left(\left|F_{x_{0}}\left(x, y_{i}\right)-F_{x_{0}}\left(x, y_{j}\right)\right|\right)=g(x, y)$,

which verifies

$$
\left|\partial_{x} g\right| \leqq\left|\partial_{x}\left(F_{x_{0}}\left(x, y_{i}\right)-F_{x_{0}}\left(x, y_{j}\right)\right)\right| \cdot\left|\widetilde{V}_{i j}^{\prime}\left(\left|F_{x_{0}}\left(x, y_{i}\right)-F_{x_{0}}\left(x, y_{j}\right)\right|\right)\right| \leqq \operatorname{const}(1+|g|),
$$

where we have combined (2.6), boundedness of $\partial_{x}\left(F_{x_{0}}(x, y)\right)$ uniformly in $y_{j}$ and (2.2). 
By induction this gives

$$
\left|\partial_{x}^{\alpha} g(x, y)\right| \leqq C_{\alpha}(1+|g(x, y)|), \quad\left(\alpha \in \mathbb{N}^{3 m}, x \in \Omega_{x_{0}}, y \in \mathbb{R}^{p}\right) .
$$

c) $V_{i j}\left(\left|A_{i} x-F_{x_{0}}\left(x, y_{j}\right)\right|\right)=V_{i j}\left(\left|F_{x_{0}}\left(x, A_{i} x_{0}\right)-F_{x_{0}}\left(x, y_{i}\right)\right|\right)=g(x, y)$

which using the same arguments also verifies the estimate (2.7).

It follows from this estimate that $V\left(x, F_{0}(x, y)\right)\left(-\Delta_{y}+1\right)^{-1} \in C^{\infty}\left(\Omega_{x_{0}}, L^{2}\left(\mathbb{R}^{p}\right)\right)$; thus hypothesis $(\mathrm{H} 2)$ is verified in some small neighborhood of any point $x_{0} \in \mathbb{R}^{3 m} \backslash \mathscr{C}$.

We shall now consider an interval $I$ of energy values such that

$$
\lambda_{1}^{-1}(I) \subset \mathbb{R}^{3 m} \text { is compact and disjoint from } \mathscr{C} \text {. }
$$

Then $\lambda_{1}^{-1}(I)$ can be covered by finitely many open sets $\Omega_{j}=\Omega_{x_{0}^{j}}$ of the above form. If in addition the separation conditions $(\mathrm{H} 3)(\mathrm{H} 4)$ on $S p(P)$ are satisfied, Theorem 1.2 is applicable and we have (with analogous notations):

Theorem 2.1. Assume (H mol), (H3) (H4). Then one has for $\lambda \in I$,

$$
\lambda \in \operatorname{Sp}\left(P^{\zeta}\right) \Leftrightarrow 0 \in \operatorname{Sp}\left(E_{-+}(\lambda)\right),
$$

where $E_{-+}(\lambda)$ is an h-admissible operator on $\bigoplus_{l=1}^{M} L^{2}\left(\mathbb{R}^{3 m}\right)$ which depends analytically on $\lambda$, with principal symbol given by (1.20). Furthermore, for $M=1$, one can choose $\zeta$ such that the scalar operator $E_{-+}(\lambda)$ corresponding to the groundstate of $Q(x)$ is O(3)-invariant, i.e. denoting by

$$
U_{R} \varphi(x)=\varphi(R \cdot x), \quad R \cdot x=\left(R x_{1}, \ldots, R x_{m}\right), \quad\left(x \in \mathbb{R}^{3 m}\right)
$$

the unitary operator on $L^{2}\left(\mathbb{R}^{3 m}\right)$ representing the orthogonal matrix $R \in O(3)$, we have

$$
\left[E_{-+}(\lambda), U_{R}\right]=0 \text {. }
$$

Proof. In view of Theorem 1.2, it remains to prove (2.10). We define by

$$
\tilde{U}_{R} \varphi(x, y)=\varphi(R \cdot x, R \cdot y), \quad \hat{U}_{R} \varphi(y)=\varphi(R \cdot y)
$$

two representations of $O(3)$ on $L^{2}\left(\mathbb{R}^{3 m} \times \mathbb{R}^{3 q}\right)$ and $L^{2}\left(\mathbb{R}^{3 q}\right)$ respectively, and set

$$
\mathscr{U}_{R}=\left[\begin{array}{cc}
\tilde{U}_{R} & 0 \\
0 & U_{R}
\end{array}\right] .
$$

Since all potentials are radial, we deduce that

$$
\hat{U}_{R}^{-1} Q(x) \hat{U}_{R}=Q\left(R^{-1} \cdot x\right),
$$

and in view of the nondegeneracy of the groundstate $u_{1}(x)$ of $Q(x)$ we get from $(2.11)$ that

$$
u_{1}(R \cdot x, R \cdot y)=u_{1}(x, y), \quad \lambda_{1}(R \cdot x)=\lambda_{1}(x) .
$$

Choosing $\zeta$ and all cut-off functions in the proof of Lemma 1.1 $O(3)$-invariant (and also the function $\left.g_{1} \in H^{2}\left(\mathbb{R}_{y}^{p}\right)\right)$, one gets $w_{1}(R x, R y)=w_{1}(x, y)$ for all $(x, y) \in \mathbb{R}^{3(m+q)}$ and $R \in O(3)$.

It follows readily that $\mathscr{P}_{\lambda}$ commutes with $\mathscr{U}_{R}$, hence does $\mathscr{E}=\mathscr{P}_{\lambda}^{-1}$, and this gives (2.10). 
Remark 2.2. In the case $M=1$ assumption (H4) can be deleted in Theorem 2.1. In fact, it then follows from (H3) that for $x$ in some neighborhood $\mathscr{M}$ of $\lambda^{-1}(I), \lambda_{1}(x)$ $=\operatorname{Inf} S p(Q(x))$ is a discrete eigenvalue. Since the range of the associated projection $\Pi(x)$ is spanned by a positive function of $y \in \mathbb{R}^{p}$, one readily gets a global continuous section $u_{1}(x, \cdot)$ trivializing the line bundle $E$ induced by $\Pi(x)$ over $\mathscr{M}$, regardless of the topology of $\mathscr{M}$. Modifying $u_{1}(x, \cdot)$ as before we obtain a Grushin problem of the form (1.17) and thus Theorem 2.1.

\section{WKB Constructions for a Diatomic Molecule}

In this section we shall consider WKB expansions for eigenfunctions and eigenvalues of a diatomic molecule with $q$ electrons near the bottom of the potential well formed by the electronic groundstate $u_{1}(x, \cdot)$ of the operator $Q(x)$ acting in $L^{2}\left(\mathbb{R}_{y}^{p}\right), p=3 q$. Thus we assume (H mol) with $m=1$ and (H3) for $M=1$ and some interval $I=(-\infty, b]$ containing $\lambda_{0}=\inf _{x \in \mathbb{R}^{3}} \lambda_{1}(x)$.

The set $\mathscr{C}$ of nuclear collisions then simply equals the origin $\{0\}$ in $\mathbb{R}^{3}$ (with the implicit condition $A_{1} \neq A_{2}$ ), and we assume that the bottom of the potential well $\Gamma=\lambda_{1}^{-1}\left(\lambda_{0}\right)$ satisfies

(H5) $\Gamma=\left\{x \in \mathbb{R}^{3} ;|x|=r_{0}\right.$ for some $r_{0}>0$, and $\left.\partial_{r}^{2} \lambda_{1}\right|_{\Gamma}>0$,

where $\partial_{r}$ denotes the radial derivative in $\mathbb{R}^{3}$. In view of (2.12) we shall denote (with the usual abuse of notation) the constant value of $\partial_{r}^{2} \lambda_{1}$ on $\Gamma$ also by $\lambda_{1}^{\prime \prime}\left(r_{0}\right)$. We write $\psi(x)$ for the distance of $x \in \mathbb{R}^{3}$ to $\Gamma$ in the degenerate metric $\left(\lambda_{1}(x)-\lambda_{0}\right) d x^{2}$, and since $\psi$ only depends on the radial variable $r=|x|$, one immediately sees that $\psi$ is $C^{\infty}$ in a neighborhood of $\Gamma$ of the form $\Omega=\left\{x \in \mathbb{R}^{3} ;|x| \in \Omega^{\prime}\right\}$, where $\Omega^{\prime}=\left(r_{0}-\varepsilon, r_{0}+\varepsilon\right)$ for some $\varepsilon>0$, and satisfies

$$
(\nabla \psi)^{2}(x)=\lambda_{1}(x)-\lambda_{0} \quad(x \in \Omega) .
$$

We remark that the only obstruction to $\psi$ being in $C^{\infty}\left(\mathbb{R}^{3}\right)$ is a possible absorption of $\lambda_{1}(x)$ into $S p_{\text {ess }}(Q(x))$ or $\lambda_{1}(x) \rightarrow \infty$.

Choosing $\Omega$ sufficiently small, we may assume that $(\mathrm{H} 3)$ holds on $\Omega$. Next we fix some (arbitrarily large) $C_{0}>0$ and denote by $e_{1}, \ldots, e_{N_{0}}$ the eigenvalues in $\left[0, C_{0}\right]$ of the 1-dimensional harmonic oscillator

$$
H_{0}=-\partial_{r}^{2}+\frac{1}{2} \lambda_{1}^{\prime \prime}\left(r_{0}\right)\left(r-r_{0}\right)^{2}
$$

i.e. $e_{j}=\psi^{\prime \prime}\left(r_{0}\right)(2 j+1)$. Then we first have the following formal result:

Theorem 3.1. Under the above assumptions $(\mathrm{H} \mathrm{mol}),(\mathrm{H} 3),(\mathrm{H} 5)$ there are approximate eigenvalues $\lambda_{j}^{l}(h) \in \mathbb{R}$ and approximate eigenfunctions $w_{j}^{l, m}(h) \in H^{2}\left(\Omega \times \mathbb{R}^{p}\right)$ such that one has for $j \in\left\{1, \ldots, N_{0}\right\}, l \in\{0,1,2, \ldots\}, m=-l, \ldots, l$ and $h>0$ sufficiently small

$$
\begin{gathered}
\lambda_{j}^{l}(h) \sim \lambda_{0}+e_{j} h+\sum_{k \geqq 2} \lambda_{j, k}^{l} h^{k}, \\
e^{\psi(x) / h} w_{j}^{l, m}(x, y ; h) \sim h^{-m_{j}}\left\{w_{j, 0}^{l, m}(x) u_{1}(x, y)+\sum_{k \geqq 1} w_{j, k}^{l, m}(x, y) h^{k / 2}\right\}
\end{gathered}
$$

as $h \downarrow 0$, where $w_{j, 0}^{l, m} \in C^{\infty}(\Omega), w_{j, k}^{l, m} \in H^{2}\left(\Omega \times \mathbb{R}^{p}\right), m_{j} \in \mathbb{R}, m_{1}=\frac{1}{4}$ and the asymptotic expansion holds in $H^{2}\left(\Omega \times \mathbb{R}^{p}\right)$,

$$
\left\langle w_{j}^{l, m}(h), w_{j^{\prime}}^{l^{\prime}, m^{\prime}}(h)\right\rangle_{L^{2}\left(\Omega \times \mathbb{R}^{p}\right)}=\delta_{j, j^{\prime}} \delta_{l, l^{\prime}} \delta_{m, m^{\prime}}+\mathcal{O}\left(h^{\infty}\right)
$$


and

$$
\left\|e^{\psi(x) / h}\left(P-\lambda_{j}^{l}(h)\right) w_{j}^{l, m}(h)\right\|_{L^{2}\left(\Omega \times \mathbb{R}^{p}\right)}=\mathcal{O}\left(h^{\infty}\right) .
$$

The main idea of the proof is to use a simplified "formal" version of Theorem 2.1 to relate the construction of WKB functions for $P$ modulo coordinate transformations to the construction of the kernel of a formal pseudodifferential operator $\widetilde{E}_{-+}(\lambda)$ which is naturally induced by the symbol of the $h$-admissible operator $E_{-+}(\lambda)$ in Theorem 2.1. This can then be accomplished as in [Ma2], and is actually much simplified by the $O(3)$-invariance which leads to absence of half-integer powers of $h$ in the eigenvalue expansion (3.1).

Before turning to the proof of Theorem 3.1, we shall therefore recall some basic facts on formal pseudodifferential operators with operator valued symbol. If $\Omega$ is an open set in $\mathbb{R}^{n}, H$ a Hilbert space and $m \in \mathbb{R}$, we introduce the space

$$
S^{m}(\Omega, H)=\left\{\sum_{k \geqq 0} h^{-m+k / 2} s_{k}(x) ; s_{k} \in C^{\infty}(\Omega, H)\right\}
$$

of formal power series. For $\psi \in C^{\infty}(\Omega, \mathbb{R})$ and $\mathscr{V}$ being a (sufficiently small) neighborhood of 0 in $\mathbb{R}^{n}$ we then set

$$
\Omega^{*}=\Omega_{\psi}^{*}=\left\{(x, \xi) \in \Omega \times \mathbb{C}^{n} ; \xi-i \nabla \psi(x) \in \mathscr{V}\right\}
$$

and

$$
S^{0}\left(\Omega^{*} ; \mathscr{L}\left(H_{1}, H_{2}\right)\right)=\left\{\sum_{k \geqq 0} k^{k} p_{k}(x, \xi) ; p_{k} \in C^{\infty}\left(\Omega^{*} ; \mathscr{L}\left(H_{1}, H_{2}\right)\right)\right\}
$$

where $H_{1}, H_{2}$ denote Hilbert spaces. For any symbol $a=a(x, \xi, h) \in S^{0}\left(\Omega^{*} ; \mathscr{L}\left(H_{1}, H_{2}\right)\right)$ one then defines an operator $a\left(x, h D_{x}, h\right)$ on $e^{-\psi(x) / h} S^{m}\left(\Omega, H_{1}\right)$ (which we shall call a formal pseudodifferential operator) by setting for $s \in S^{m}\left(\Omega, H_{1}\right)$,

$$
\begin{gathered}
a\left(x, h D_{x}, h\right)\left(e^{-\psi(x) / h} s(x, h)\right) \\
=e^{-\psi(x) / h} \sum_{\alpha \in \mathbb{N}^{n}} \frac{h^{|\alpha|}}{i^{|\alpha|} \alpha !} \partial_{\xi}^{\alpha} a(x, i \nabla \psi(x), h) \partial_{x^{\prime}}^{\alpha}\left(s\left(x^{\prime}, h\right) e^{\kappa\left(x, x^{\prime}\right) / h}\right)_{x^{\prime}=x},
\end{gathered}
$$

where

$$
\kappa\left(x, x^{\prime}\right)=\psi\left(x^{\prime}\right)-\psi(x)-\left(x^{\prime}-x\right) \cdot \nabla \psi(x)=\mathcal{O}\left(\left|x-x^{\prime}\right|^{2}\right) .
$$

Formula (3.7) coincides with a formal stationary phase expansion, if the usual (mathematical) quantization convention is used for $a\left(x, h D_{x}\right)$. We remark that as a trivial consequence $O(n)$-invariance of the symbol, i.e. $a(R x, R \xi, h)=a(x, \xi, h)$ for all $R \in O(n)$, implies $\left[a\left(x, h D_{x}, h\right), U_{R}\right]=0$. Next one verifies as in [Ma2] that

$$
e^{\psi(x) / h} a\left(x, h D_{x}, h\right) e^{-\psi(x) / h} S(x, h) \in S^{m}\left(\Omega, H_{1}\right) .
$$

Furthermore such operators can be composed if domain and range of the operator-valued symbols match, and one has

$$
a\left(x, h D_{x}, h\right) \circ b\left(x, h D_{x}, h\right)=(a \# b)\left(x, h D_{x}, h\right)
$$

with $a \# b$ given by the usual formula (1.18). 
Proof of Theorem 3.1. As in Sect. 2, we cover $\bar{\Omega}$ by finitely many open sets $\Omega_{j}=\Omega_{x_{j}}$ containing the point $x_{j} \in \mathbb{R}^{3}, j=1, \ldots, r$, such that the transformation

$$
F_{j}(x, y)=\left(F_{x_{j}}\left(x, y_{1}\right), \ldots, F_{x_{j}}\left(x, y_{q}\right)\right), \quad\left(x \in \Omega_{j}, y \in \mathbb{R}^{3 q}\right)
$$

satisfies (H2); here $F_{x_{j}}$ is defined by (2.3).

We set

$$
\mathscr{P}_{\lambda}=\left[\begin{array}{cc}
P-\lambda & u_{1} \\
\left\langle\cdot, u_{1}\right\rangle_{y} & 0
\end{array}\right]
$$

and recall that

$$
\mathscr{U}_{j}=\left[\begin{array}{cc}
U_{j} & 0 \\
0 & 1
\end{array}\right]
$$

is a well defined unitary map on $L^{2}\left(\Omega_{j}, L^{2}\left(\mathbb{R}^{p}\right) \oplus \mathbb{C}\right)$. Using formula (1.22) one readily sees that $U_{j} P U_{j}^{-1}$ induces a formal pseudodifferential operator with symbol $p_{j}(x, \xi) \in S^{0}\left(\Omega_{j}^{*}, \mathscr{L}\left(H^{2}\left(\mathbb{R}^{p}\right), L^{2}\left(\mathbb{R}^{p}\right)\right)\right)$, where $\Omega_{j}^{*}$ is defined as in (3.6) with $\psi(x)$ being the Agmon distance; hence $\widehat{\mathscr{P}}_{j}=\mathscr{U}_{j} \mathscr{P}_{\lambda} \mathscr{U}_{j}^{-1}$ is a formal PDO with symbol

$$
\widehat{\mathscr{P}}_{j}(x, \xi ; h)=\left[\begin{array}{cc}
p_{j}(x, \xi)-\lambda & u_{1, j}(x) \\
\left\langle\cdot, u_{1, j}(x)\right\rangle_{y} & 0
\end{array}\right],
$$

where $u_{1, j}(x)=U_{j}(x) u_{1}(x) \in C^{\infty}\left(\Omega_{j}, H^{2}\left(\mathbb{R}^{p}\right)\right)$.

We shall now relate the construction of an inverse $\widehat{\mathscr{E}}_{j}=\widehat{\mathscr{P}}_{j}^{-1}$ to the construction in the proof of Theorem 1.2. Without loss of generality we may assume that the cut-off function $\zeta$ equals 1 on the set $\Omega$ considered here, and that $w_{1}=u_{1}$ on $\Omega$. Then $\mathscr{P}_{\lambda}$ given by (1.17) coincides with $\mathscr{P}_{\lambda}$ given by (3.9) on $\Omega$, and for any $x_{0} \in \Omega_{j}$ there is $\chi_{j} \in C_{0}^{\infty}\left(\Omega_{j}\right)$ such that the symbol $\sigma\left(\mathscr{P}_{j}\right)$ of $\mathscr{U}_{j} \chi_{j} \mathscr{P}_{\lambda} \mathscr{U}_{j}^{-1} \chi_{j}$ coincides with the symbol of $\widehat{\mathscr{P}}_{j}$ in some neighborhood of $x_{0}$. Furthermore both $\sigma\left(\mathscr{P}_{j}\right)$ and $\sigma\left(\widehat{\mathscr{P}}_{j}\right)$ are analytic in $\xi \in \mathbb{C}^{3}$; it follows readily from the symbolic calculus that the symbol of $\widetilde{\mathscr{E}}_{j}=\mathscr{U}_{j} \chi_{j} \mathscr{E} \mathscr{U}_{j}^{-1} \chi_{j}$ extends analytically in $\xi$ to $\Omega^{*}$.

Thus, setting $\widehat{\mathscr{E}}_{j}(x, \xi, h)=\sigma\left(\widetilde{\mathscr{E}}_{j}\right)(x, \xi, h)$ for $x$ close to $x_{0}$ and $\chi_{j}$ as above, we have determined a unique symbol in $S^{0}\left(\Omega_{j}^{*}, \mathscr{L}\left(H^{2}\left(\mathbb{R}^{p}\right) \oplus \mathbb{C}, L^{2}\left(\mathbb{R}^{p}\right) \oplus \mathbb{C}\right)\right)$, which in view of (1.40) satisfies

$$
\begin{aligned}
& \left(\widehat{\mathscr{P}}_{j} \# \widehat{\mathscr{C}}_{j}\right)(x, \xi, h)=\mathbf{1}_{L^{2}\left(\mathbb{R}^{p}\right) \oplus \mathbb{C}}, \\
& \left(\widehat{\mathscr{C}}_{j} \# \widehat{\mathscr{P}}_{j}\right)(x, \xi, h)=\mathbf{1}_{H^{2}\left(\mathbb{R}^{p}\right) \oplus \mathbb{C}},
\end{aligned}
$$

for all $(x, \xi) \in \Omega_{j}^{*}$. We remark that $\widehat{\mathscr{E}}_{j}(x, \xi, h)$ can of course be constructed directly from (3.10), and since the calculus of formal PDO is completely local in $x$, this in fact is easier than the construction in the proof of Theorem 1.2. Anyhow, writing

$$
\widehat{\mathscr{E}}_{j}=\left[\begin{array}{cc}
\hat{E}_{j} & \hat{E}_{+, j} \\
\hat{E}_{-, j} & \hat{E}_{-+, j}
\end{array}\right]
$$

for the associated formal PDO, we have

$$
\widehat{\mathscr{E}}_{l}=\mathscr{U}_{l} \mathscr{U}_{j}^{-1} \hat{\mathscr{E}}_{j} \mathscr{U}_{j} \mathscr{U}_{l}^{-1} \text { in } \Omega_{j} \cap \Omega_{l}
$$

since $\widehat{\mathscr{P}}_{\lambda}=\mathscr{U}_{l} \mathscr{U}_{j}^{-1} \widehat{\mathscr{P}}_{j} \mathscr{U}_{j} \mathscr{U}_{l}^{-1}$; it follows that

$$
\hat{E}_{-+, j}=\hat{E}_{-+, l} \text { in } \Omega_{j} \cap \Omega_{l},
$$


and thus all $\hat{E}_{-+, j}, j=1, \ldots, r$, patch together to define a global formal PDO $\hat{E}_{-+}(\lambda)$ of class $S^{0}\left(\Omega^{*} ; \mathscr{L}(\mathbb{C}, \mathscr{C})\right)$ with principal symbol $\lambda-\xi^{2}-\lambda_{1}(x)$. By construction of $\hat{\mathscr{E}}_{j}$ the symbol of $\hat{E}_{-+}(\lambda)$ coincides with the symbol of the $h$-admissible operator $E_{-+}(\lambda)$ in Theorem 2.1 (using analytic continuation in $\xi$ ), which gives

$$
\left[\hat{E}_{-+}(\lambda), U_{R}\right]=0 \quad \text { on } \quad e^{-\psi(x) / h} S^{m}(\Omega, \mathbb{C}) \text { for all } R \in O(3)
$$

Denoting by $Y_{l}^{m}$ (a real choice of) the spherical harmonics, $\mathscr{H}_{l}=\operatorname{Span}\left\{Y_{l}^{m} ;-1\right.$ $\leqq m \leqq l\}$ carries an irreducible representation of $O(3)$. It follows from (3.13) that one has the decomposition

$$
\hat{E}_{-+}(\lambda)=\bigoplus_{l=0}^{\infty} E_{-+}^{l}(\lambda) \otimes \mathbf{1}_{\mathscr{H}_{l}}
$$

where for any symbol $\alpha \in e^{-\psi(r) / h} S^{m}\left(\Omega^{\prime}, \mathbb{C}\right)$ depending only on the radial variable $r$

$$
E_{-+}^{l}(\lambda) \alpha \delta_{m, m^{\prime}}=\left\langle\hat{E}_{-+}(\lambda) \alpha Y_{l}^{m}, Y_{l}^{m^{\prime}}\right\rangle_{L^{2}\left(S^{2}\right)}
$$

(use Schur's lemma). Furthermore the principal symbol of $E_{-+}^{l}(\lambda)$ is $\lambda-\varrho^{2}-\lambda_{1}(r)$, where $\varrho$ denotes the dual variable for $r$.

We shall now construct the formal solutions of $\hat{E}_{-+}(\lambda) u=0$, which will then lead to the WKB functions of Theorem 3.1 for $P$. Since $E_{-+}^{l}(\lambda)$ is formally selfadjoint for the measure $r^{2} d r$ and $\lambda_{1}$ as a function of $r$ admits a nondegenerate minimum in $r_{0}$, the construction of Helffer and Sjöstrand in [HS1] Sect. 3 gives $N_{0}$ formal series of the form

$$
\begin{gathered}
\alpha_{j}^{l}(r, h ; \lambda)=e^{-\psi(r) / h} \sum_{k \geqq 0} \alpha_{j, k}^{l}(r ; \lambda) h^{k / 2-m_{j}}, \\
\left(\alpha_{j, k}^{l}(\cdot ; \lambda) \in C^{\infty}\left(\Omega^{\prime}, \mathbb{R}\right), m_{j} \in \mathbb{R}, 1 \leqq j \leqq N_{0}\right)
\end{gathered}
$$

associated with the formal eigenvalues

$$
\mu_{j}^{l}(h ; \lambda)=\lambda_{0}+e_{j} h+\sum_{k \geqq 2} \mu_{j, k}^{l}(\lambda) h^{k}
$$

such that

$$
\begin{gathered}
E_{-+}^{l}(\lambda) \alpha_{j}^{l}(r, h ; \lambda)=\left(\lambda-\mu_{j}^{l}(h ; \lambda)\right) \alpha_{j}^{l}(r, h ; \lambda) \quad \text { in } e^{-\psi(r) / h} S^{m_{j}}\left(\Omega^{\prime}\right), \\
\left\langle\alpha_{j}^{l}(\cdot, h ; \lambda), \alpha_{j^{\prime}}^{l}(\cdot, h ; \lambda)\right\rangle=\delta_{j j^{\prime}},
\end{gathered}
$$

where (3.19) holds in the sense of formal power series in $h$ with complex coefficients and the inner product $\langle\cdot, \cdot\rangle$ is defined by a formal stationary phase expansion at $r_{0}$.

We remark that since the eigenvalues $e_{j} h$ of the harmonic approximation are simple, half-integer powers of $h$ are absent in (3.17) and, furthermore, the coefficients $\mu_{j, k}^{l}(\lambda)$ - and $\alpha_{j, k}^{l}(r ; \lambda)$ - depend analytically on $\lambda$ (write the spectral projection in terms of the resolvent of $\left.E_{-+}^{l}(\lambda)-\lambda\right)$. Thus the zeroes of

$$
\lambda=\mu_{j}^{l}(h, \lambda)
$$

in the space of formal power series are simply given by the Lagrange inversion formula

$$
\lambda_{j}^{l}(h)=\lambda_{0}+\sum_{k \geqq 1} h^{k} \frac{1}{k !} \partial_{\lambda}^{k-1}\left\{e_{j}+\sum_{n \geqq 2} \mu_{j, n}^{l}(\lambda) h^{n-1}\right\}^{k}\left(\lambda=\lambda_{0}\right)=\lambda_{0}+e_{j} h+\sum_{k \geqq 2} \lambda_{j, k}^{l} h^{k} .
$$


Thus, setting

$$
\alpha_{j}^{l}(r, h)=\alpha_{j}^{l}\left(r, h ; \lambda=\lambda_{j}^{l}(h)\right)
$$

and using analyticity of $\alpha_{j, k}^{l}(r, \lambda)$ in $\lambda$, we have found formal series which satisfy

$$
E_{-+}^{l}\left(\lambda_{j}^{l}(h)\right) \alpha_{j}^{l}(r, h)=0 \quad \text { in } \quad e^{-\psi(r) / h} S^{m_{j}}\left(\Omega^{\prime}\right) .
$$

In view of (3.14)

$$
\beta_{j}^{l, m}(x, h)=\alpha_{j}^{l}(|x|, h) Y_{l}^{m}\left(\frac{x}{|x|}\right), \quad(-l \leqq m \leqq l),
$$

then solve

$$
\widehat{E}_{-+}\left(\lambda_{j}^{l}(h)\right) \beta_{j}^{l, m}=0 \quad \text { in } \quad e^{-\psi(x) / h} S^{m_{j}}(\Omega),
$$

and

$$
\left\langle\beta_{j}^{l, m}, \beta_{j^{\prime}}^{l^{\prime}, m^{\prime}}\right\rangle_{L^{2}(\Omega)}=\delta_{j j^{\prime}} \delta_{l l^{\prime}} \delta_{m m^{\prime}}
$$

with a formal inner product as in (3.19). Since it follows from (3.11) that

$$
\widehat{\mathscr{P}}_{k} \circ \widehat{\mathscr{E}}_{k}=1 \text { in } e^{-\psi(x) / h} S^{m}\left(\Omega_{k}, L^{2}\left(\mathbb{R}^{p}\right) \oplus \mathbb{C}\right),
$$

Eq. (3.24) yields by definition of $\widehat{\mathscr{P}}_{k}$, $\widehat{\mathscr{E}}_{k}$ that the formal symbol

$$
{ }_{k} \tilde{v}_{j}^{l, m}=E_{+, k}\left(\lambda_{j}^{l}(h)\right) \beta_{j}^{l, m} \quad \text { in } \quad e^{-\psi(x) / h} S^{m}\left(\Omega_{k}, H^{2}\left(\mathbb{R}^{p}\right)\right)
$$

solves

$$
\left(U_{k} P U_{k}^{-1}-\mu_{j}^{l}(h)\right)_{k} \tilde{v}_{j}^{l, m}=0 \quad \text { in } \quad e^{-\psi(x) / h} S^{m}\left(\Omega_{k}, L^{2}\left(\mathbb{R}^{p}\right)\right)
$$

Since

$$
E_{+, k}(\lambda)=U_{k}(x) u_{1}(x, \cdot)+\mathcal{O}(h) \text { and }\left\langle u_{1}(x), u_{1}(x)\right\rangle_{y}=1,
$$

we get

$$
\left\langle\tilde{v}_{j}^{l, m},{ }_{k} \tilde{v}_{j^{\prime}}^{l^{\prime}, m^{\prime}}\right\rangle_{L^{2}\left(\Omega_{k} \times \mathbb{R}^{p}\right)}=\delta_{j j^{\prime}} \delta_{l l^{\prime}} \delta_{m m^{\prime}}+\mathcal{O}(h) .
$$

In view of (3.12) we have

$$
\begin{array}{ll} 
& U_{i}^{-1}{ }_{i} \tilde{v}_{j}^{l, m}=U_{k}^{-1}{ }_{k} \tilde{v}_{j}^{l, m} \text { in } \Omega_{i} \cap \Omega_{k} \\
\text { for } \quad & 1 \leqq i, k \leqq r, 1 \leqq j \leqq N_{0}, l \in \mathbb{N},-l \leqq m \leqq l .
\end{array}
$$

Thus the $U_{k}^{-1}{ }_{k} \tilde{v}_{j}^{l, m}$ patch together to define globally in $x \in \Omega$ a formal symbol $\tilde{w}_{j}^{l, m}$ with coefficients in $H^{2}\left(\Omega \times \mathbb{R}^{p}\right)$. Resumming the formal series ${ }_{k} \tilde{v}_{j}^{l, m}, \tilde{w}_{j}^{l, m}$ (and $\lambda_{j}^{l}(h)$ ) with the same Borel-procedure, we obtain functions ${ }_{k} v_{j}^{l, m}(h) \in C^{\infty}\left(\Omega_{k}, H^{2}\left(\mathbb{R}^{p}\right)\right)$, $w_{j}^{l, m}(h) \in H^{2}\left(\Omega \times \mathbb{R}^{p}\right)$ which depend smoothly on $h$ and are related by

$$
U_{k}(x) w_{j}^{l, m}(x, \cdot ; h)={ }_{k} v_{j}^{l, m}(x, \cdot ; h), \quad\left(x \in \Omega_{k}\right) .
$$

The $w_{j}^{l, m}(x, y)$ now yield functions verifying all assertions of Theorem 3.1:

The asymptotic form (3.2) is an immediate consequence of (3.29), (3.27) and the smoothness of $\beta_{j}^{l, m}$ in $x \in \Omega$, and (3.4) follows combining (3.32) and (3.28) with a remainder estimate on the stationary phase expansion.

To prove (3.3), note that in view of (3.30),

$$
\left\langle w_{j}^{l, m}, w_{j^{\prime}}^{l^{\prime}, m^{\prime}}\right\rangle_{L^{2}\left(\Omega \times \mathbb{R}^{p}\right)}=\delta_{j j^{\prime}} \delta_{l l^{\prime}} \delta_{m m^{\prime}}+\mathcal{O}(h)
$$


and

$$
\left(\mu_{j}^{l}(h)-\mu_{j^{\prime}}^{l^{\prime}}(h)\right)\left\langle w_{j}^{l, m}, w_{j^{\prime}}^{l^{\prime}, m^{\prime}}\right\rangle_{L^{2}\left(\Omega \times \mathbb{P}^{p}\right)}=\mathcal{O}\left(h^{\infty}\right),
$$

since $P$ is self-adjoint. Equation (3.34) shows that eigenfunctions associated with different eigenvalues already are orthonormalized mod $O\left(h^{\infty}\right)$, while (3.33) allows to orthonormalize those eigenfunctions which correspond to the same formal eigenvalue $\lambda_{j}^{l}(h)$. Since this orthonormalization neither changes (3.4) nor the first term in the asymptotic form (3.2), the proof of Theorem 3.1 is complete.

Now, we are going to give a justification of the formal constructions above, in the following Theorem 3.2. First of all, let us state some basic facts. By Proposition 1.5 , if one takes $c_{1}>0$ arbitrarily large such that:

$$
\lambda_{0}+e_{1} h+c_{1} h^{2} \notin S p\left(P_{0}\right),
$$

one can see that the number:

$$
N_{1}=\# \operatorname{Sp}(P) \cap\left(-\infty, \lambda_{0}+e_{1} h+c_{1} h^{2}\right]=\# \operatorname{Sp}\left(P_{0}\right) \cap\left(-\infty, \lambda_{0}+e_{1} h+c_{1} h^{2}\right]
$$

is independent of $h>0$ small enough, due also to the explicit expansions of the low eigenvalues of $P_{0}$ under the assumption of Theorem 3.1. Then we have:

Theorem 3.2. Under the assumptions of Theorem 3.1, the eigenvalues $E_{1}(h), \ldots, E_{N_{1}}(h)$ of $P$ in $\left(-\infty, \lambda_{0}+e_{1} h+c_{1} h^{2}\right]$ admit asymptotic expansions:

$$
E_{j}(h)=e_{1} h+\sum_{k \geqq 2} e_{j, k} h^{k} \quad(h \rightarrow \infty),
$$

and if $E_{j}(h)$ is asymptotically simple (in the sense that the expansion (3.36) determines $E_{j}(h)$ in a unique way), the associated normalized eigenfunction $v_{j}$ satisfies:

$$
e^{\psi(x) / h} v_{j}(x, y ; h) \sim h^{-1 / 4}\left(v_{j, 0}(x) u_{1}(x, y)+\sum_{k \geqq 1} v_{j, k}(x, y) h^{k / 2}\right), \bullet
$$

where $v_{j, 0} \in C^{\infty}(\Omega), v_{j, k}(x, y) \in H^{2}\left(\Omega \times \mathbb{R}^{p}\right)$, and the expansion holds in $H^{1}\left(\Omega ; L^{2}\left(\mathbb{R}^{p}\right)\right)$.

Proof. Thanks to Proposition 1.5, we see that there is a gap of order of magnitude $h^{2}$ between the eigenvalues of $P$ in $I(h)=\left(-\infty, \lambda_{0}+e_{1} h+c_{1} h^{2}\right]$ and the other ones. Now, let $\chi \in C_{0}^{\infty}(\Omega), \chi=1$ near $\Gamma$, and let $\widetilde{F}$ be the space generated by all the $\chi(x) w_{1}^{l, m}(x, y ; h)$ such that $\lambda_{1}^{l}(h) \in I(h)$ (cf. Theorem 3.1). Let also $\mathscr{F}$ be the eigenspace of $P$ associated to the eigenvalues in $I(h)$. We can then apply Proposition 2.5 of [HS1], and we obtain:

$$
\sup _{\substack{v \in \mathscr{F} \\\|v\|=1}} d(v, \mathscr{F})=\mathcal{O}\left(h^{\infty}\right)
$$

where $d(v, \mathscr{F})=\inf _{w \in \mathscr{F}}\|v-w\|_{L^{2}\left(\mathbb{R}^{3} \times \mathbb{R}^{p}\right)}$. This proposition also proves the fact that the eigenvalues of $P$ admit the asymptotic expansions given in (3.1) with $j=1$. Then, using the Agmon-type inequality:

$$
\left\langle e^{\phi(x) / h} P e^{-\phi(x) / h} u, u\right\rangle \geqq h^{2}\left\|\nabla_{x} u\right\|+\left\langle\left(\lambda_{1}(x)-(\nabla \phi)^{2} u, u\right\rangle,\right.
$$

and following the proof of [HS1, Theorem 5.8], we see that the estimate (3.37) can be improved far from the well, and gives:

$$
\left\|e^{\psi(x) / h}\left(v_{j}-v_{j}^{*}\right)\right\|_{H^{1}\left(K ; L^{2}\left(\mathbb{R}^{p}\right)\right)}=\mathcal{O}\left(h^{\infty}\right)
$$


with $K=\{\chi=1\}$ and $v_{j}^{*}=\sum_{j, k} c_{j, k}(h) w_{1}^{k}$, where $\left(c_{j, k}\right)_{1 \leqq j, k \leqq N_{1}}$ is an orthogonal matrix, and $w_{1}^{k}$ stands for $w_{1}^{l, m}$ constructed in Theorem 3.1. The estimate (3.39) permits to end the proof easily.

\section{Polyatomic Molecules}

In this section we shall derive WKB expansions for more than 3 nuclei, i.e. we assume $(\mathrm{H} \mathrm{mol})$ with $m \geqq 2$, and suppose in addition that $\lambda_{1}(x)$ is a discrete eigenvalue of $Q(x)$ in a neighborhood of $\lambda_{0}=\operatorname{Inf}_{x \in \mathbb{R}^{3 m}} \lambda_{1}(x)$. We only discuss the groundstate and, in the case of a non-planar molecule (to be defined below), the splitting of the groundstate due to tunneling. We remark that our methods can be generalized to yield results on higher eigenvalues also.

We shall assume in analogy to Sect. 3 , that the bottom of the potential well $\Gamma=\lambda_{1}^{-1}\left(\lambda_{0}\right)$ of the effective Hamiltonian is minimal in the following sense

(H6) $\Gamma=\left\{R \cdot x_{0} ; R \in O(3)\right\}$ for some $x_{0}=\left(x_{1}^{0}, \ldots, x_{m}^{0}\right) \in \mathbb{R}^{3 m} \backslash \mathscr{C}$,

where

$$
R \cdot x_{0}=\left(R x_{1}^{0}, \ldots, R x_{m}^{0}\right) .
$$

More precisely, we see that $\Gamma$ prescribes the shape of the molecule. In fact, its geometric structure is determined by the vectorspace $E=\operatorname{Span}\left\{x_{1}^{0}, \ldots, x_{m}^{0}\right\} \subset \mathbb{R}^{3}$. Recalling that $x_{0}$ corresponds to Jacobi-coordinates for $m+1$ nuclei in the center of mass frame, we distinguish the following three cases

Proposition 4.1. The molecule is called linear, planar, non-planar, if $\operatorname{dim} E$ equals 1 , 2,3 respectively. Then the potential well $\Gamma$ is diffeomorphic to $S^{2}, S O(3)$ and $O(3)$ respectively.

Proof. If the molecule is linear, then there is $\omega_{0} \in S^{2}$ such that $x_{j}^{0}=c_{j} \omega_{0}$ for some $c_{j} \in \mathbb{R}, j=1, \ldots, m$. Thus

$$
S_{\ni}^{2} \omega \mapsto\left(c_{1} \omega, \ldots, c_{m} \omega\right) \in \Gamma
$$

gives the required diffeomorphism. If the molecule is planar, we may suppose that $x_{1}^{0}$ and $x_{2}^{0}$ are linearly independent. Denoting by $I_{j}=\left\{R \in O(3) ; R x_{j}^{0}=x_{j}^{0}\right\}$ the isotropy subgroup of $x_{j}^{0}$, we have $I_{1} \cap I_{2}=\left\{1, R_{0}\right\}$ where $R_{0}$ is the reflection with respect to the hyperplane spanned by $x_{1}^{0}$ and $x_{2}^{0}$. Since det $R_{0}=-1, O(3) /\left\{1, R_{0}\right\}$ is diffeomorphic to $S O(3)$ and, furthermore, it acts freely on $x_{0}$ : If $R_{1} \cdot x_{0}=R_{2} \cdot x_{0}$ for some $R_{1}, R_{2} \in O(3)$, then $R_{1} x_{j}^{0}=R_{2} x_{j}^{0} \quad(j=1,2)$, which gives $R_{1}^{-1} R_{2} \in I_{1} \cap I_{2}$ $=\left\{1, R_{0}\right\}$. This yields $\Gamma \simeq O(3) /\left\{1, R_{0}\right\} \simeq S O(3)$. Finally, if the molecule is nonplanar, we suppose $x_{j}^{0}, j=1,2,3$, to be linearly independent and get $I_{1} \cap I_{2}$ $\cap I_{3}=\{1\}$ for the intersection of all isotropy subgroups. Thus $O(3)$ acts freely on $x_{0}$, proving $\Gamma \simeq O(3)$.

We remark that, since $O(3)$ consists of two connected components $O^{ \pm}(3)=\{R \in O(3) ; \operatorname{det} R= \pm 1\}$, the potential well $\Gamma$ of a non-planar molecule is a $C^{\infty}$-manifold consisting of two connected components which are interchanged by the reflection $x \mapsto-x$ in $\mathbb{R}^{3 m}$. Since for 4 or more nuclei there certainly exist many non-planar physical molecules, one is in a physically natural way lead to the problem of estimating the splitting between the eigenvalues of the initial molecular 
Hamiltonian $P$. We set $v=\operatorname{dim} \Gamma$ and denote by $\Gamma_{1}$ one of the connected components $\left(\Gamma_{1}=\Gamma\right.$ for a linear or planar molecule). We assume that the transversal Hessian of $\lambda_{1}(x)$ is of maximal rank $3 m-v$; on $\Gamma_{1}$. Denoting by $\psi(x)$ the distance between a point $x \in \mathbb{R}^{3 m}$ and $\Gamma_{1}$ in the Agmon metric $\left(\lambda_{1}(x)-\lambda_{0}\right) d x^{2}$, it is then known that there is a neighborhood $\Omega$ of $\Gamma_{1}$ such that $\psi \in C^{\infty}(\Omega)$ (see [HS2]). In our situation one can choose $\Omega$ to be invariant under the action of the group $S O(3)=O^{+}(3)$. Then the $O(3)$-invariance of the effective potential gives

$$
\psi(R \cdot x)=\psi(x), \quad(R \in S O(3), x \in \Omega) .
$$

Since $\Gamma$ is a minimal well in the sense of (H6), it follows from (4.1) that

$$
\Delta \psi(x) \text { is a constant } \mu_{1} \text { on } \Gamma_{1} .
$$

Thus the submanifold $\Gamma_{1}$ is a uniformly degenerate potential well for the effective Hamiltonian in the sense of Helffer-Sjöstrand [HS2]. One can therefore apply their results to construct the first eigenfunction. We shall, however, describe in the following a construction which we think is more direct and profits from rotational invariance. As a first step we have in analogy to Theorem 3.1

Theorem 4.2. Under the above assumptions there is an approximate eigenvalue $\mu(h) \in \mathbb{R}$ and an approximate eigenfunction $w(h)$ such that

$$
\begin{gathered}
\mu(h) \sim \sum_{j \geqq 0} \mu_{j} h^{j} ; \quad \mu_{0}=\lambda_{0}, \mu_{1}=\left.\Delta \psi(x)\right|_{\Gamma_{1}}, \\
e^{\psi(x) / h} w(x, y ; h) \sim h^{-(3 m-v) / 4} \sum_{j} a_{j}(x, y) h^{j}
\end{gathered}
$$

as $h \downarrow 0$, where $a_{j} \in H^{2}\left(\Omega \times \mathbb{R}^{p}\right)$, the expansion holds in $H^{2}\left(\Omega \times \mathbb{R}^{p}\right)$, and

$$
\left\|e^{\psi(x) / h}(P-\lambda(h)) w(h)\right\|=\mathcal{O}\left(h^{\infty}\right) .
$$

Furthermore we obtain for the first terms in the asymptotic expansion:

$$
a_{0}(x, y)=b_{0}(x) u_{1}(x, y),
$$

where $b_{0} \in C^{\infty}(\Omega)$ is the solution of the Cauchy problem

$$
\left\{\begin{array}{l}
2 \nabla \psi \cdot \nabla b_{0}+\left(\Delta \psi-\mu_{1}\right) b_{0}=0 \quad \text { in } \Omega \\
\left.b_{0}\right|_{\Gamma_{1}}=1
\end{array}\right.
$$

and

$$
\mu_{2}=\left.\left\langle\left(-\Delta_{x}+p\left(\partial_{y}\right)\right) u_{1}(x, \cdot), u_{1}(x, \cdot)\right\rangle_{y}\right|_{\Gamma_{1}}+c_{\Gamma}
$$

where

$$
c_{\Gamma}=-\left.\Delta b_{0}\right|_{\Gamma_{1}}=\left.\frac{1}{4 \mu_{1}} \Delta^{2} \psi\right|_{\Gamma_{1}}-\left.\frac{1}{2 \mu_{1}}\left|\nabla^{3} \psi\right|_{\Gamma_{1}}\right|^{2} .
$$

Remark 4.3. i) By $S O(3)$-invariance of $\Delta_{x}, p\left(\partial_{y}\right) u_{1}(x, \cdot)$ and $\psi, \mu_{2}$ is well defined by (4.8). The second equality in (4.9) follows from the first by twice differentiating (4.7) and using $\nabla \psi=0$ on $\Gamma_{1}$. We remark that the methods of Helffer-Sjöstrand naturally lead to an (identical) representation of $c_{\Gamma}$ in the form

$$
c_{\Gamma}=\left(-|\nabla c|^{2}+\Delta c\right)_{\Gamma_{1}}
$$


where $c$ solves

$$
2 \nabla \psi \nabla c-\Delta \psi+\mu_{1}=0,\left.\quad c\right|_{\Gamma_{1}}=0 .
$$

ii) For a non-planar molecule (exhibiting a symmetric double well), the reflected function $\tilde{w}(x, y ; h)=w(-x,-y ; h)$ gives a different approximate eigenfunction with energy $\mu(h)$, concentrated in the well $\Gamma_{2}=-\Gamma_{1}$.

iii) The factor $h^{-(3 m-v) / 4}$ in (4.4) is just a normalizing constant, ensuring that $\|w(h)\|=$ const $+\mathcal{O}\left(h^{1 / 2}\right)$.

Proof of Theorem 4.2. This is almost identical with the proof of Theorem 3.1. We again cover $\bar{\Omega}$ by $r$ sufficiently small open sets $\Omega_{j}$ such that on each $\Omega_{j}$, the coordinate transformation $U_{j}(x)$ introduced in Sect. 2 is well defined. Without loss we may assume that the groundstate $u_{1}(x, \cdot)$ of $Q(x)$ is well defined in $\cup \Omega_{j}$, and thus we consider the formal Grushin operator

$$
\mathscr{P}_{j}(\lambda)=\left[\begin{array}{cc}
U_{j} P U_{j}^{-1}-\lambda & u_{1, j} \\
\left\langle\cdot, u_{1, j}\right\rangle_{y} & 0
\end{array}\right] ; \quad u_{1, j}(x)=U_{j}(x) u_{1}(x, \cdot)
$$

which maps formal symbols of class $e^{-\psi(x) / h} S^{m}\left(\Omega_{j}, H^{2}\left(\mathbb{R}^{p}\right) \oplus \mathbb{C}\right)$ into $e^{-\psi(x) / h} S^{m}\left(\Omega_{j}, L^{2}\left(\mathbb{R}^{p}\right) \oplus \mathbb{C}\right)($ for each $m \in \mathbb{R})$. Here the symbol space $S^{m}\left(\Omega_{j}, H\right), H$ a Hilbert space, is defined by dropping all half-integer powers of $h$ in definition (3.5).

For notational convenience we henceforth drop the superscript " wh" which we used in Sect. 3 to distinguish formal from $h$-admissible operators. As in Sect. 3 we then get in the above symbol spaces a formal inverse

$$
\mathscr{E}_{j}(\lambda)=\left[\begin{array}{cc}
E_{j}(\lambda) & E_{j}^{+}(\lambda) \\
E_{j}^{-}(\lambda) & E_{j}^{-+}(\lambda)
\end{array}\right], \quad j=1, \ldots, r,
$$

of $\mathscr{P}_{j}$ by constructing its symbol which satisfies (3.11).

It follows from the compatibility condition

$$
\mathscr{E}_{l}=\mathscr{U}_{l} \mathscr{U}_{j}^{-1} \mathscr{E}_{j} \mathscr{U}_{j} \mathscr{U}_{l}^{-1} \text { in } \Omega_{j} \cap \Omega_{l},
$$

that the $E_{j}^{-+}(\lambda)$ patch together to define a global formal PDO $E_{-+}(\lambda)$ which is $S O(3)$-invariant.

By construction of $\mathscr{E}_{j}(\lambda), E_{j}^{ \pm}(\lambda)$ and $E_{j}^{-+}(\lambda)$ are formal $h$-pseudodifferential operators with symbols being analytic in $\xi$ near $-i \nabla \psi(x)$ and in $\lambda$ near $\lambda_{0}$; a straightforward calculation gives

$$
\begin{gathered}
\sigma\left(E_{j}^{+}(\lambda)\right)=u_{1, j}(x, \cdot)+\mathcal{O}(h), \\
\sigma\left(E_{-+}(\lambda)\right)=\lambda-\xi^{2}-\lambda_{1}(x)-h^{2}\left\{c_{1}(x)+\sum_{1 \leqq i, j \leqq 3 m} \xi_{i} \xi_{j} c_{i j}(x, \xi)\right\}+\mathcal{O}\left(h^{3}\right),
\end{gathered}
$$

where

$$
c_{1}(x)=\left\langle\left(-\Delta_{x}+p\left(\partial_{y}\right)\right) u_{1}(x, \cdot), u_{1}(x, \cdot)\right\rangle_{y},
$$

and

$$
c_{i j}(x, \xi)=\left\langle\partial_{x_{i}} X(x, \xi) \partial_{x_{j}} u_{1}(x, \cdot), u_{1}(x, \cdot)\right\rangle_{y}
$$

is a symbol of order -2 in $\xi$.

To terminate the proof, we need 
Proposition 4.4. There is a formal eigenvalue $\mu(h)$ as in Theorem 4.2 and a formal symbol

$$
\beta(x, h)=e^{-\psi(x) / h} h^{-(3 m-v) / 4} \sum_{j \geqq 0} b_{j}(x) h^{j},
$$

with $b_{j} \in C^{\infty}(\Omega)$ and $b_{0}$ given by (4.7) such that

$$
E_{-+}(\mu(h)) \beta(x, h)=0 \quad \text { in } \quad e^{-\psi(x) / h} S^{(3 m-v) / 4}(\Omega), \quad\|\beta(\cdot, h)\|=1 .
$$

Setting

$$
w(h)=U_{j}^{-1}(x) E_{j}^{+}(\mu(h)) \beta(h),
$$

and using (4.11), Theorem 4.2 follows from Proposition 4.4 as in the proof of Theorem 3.2 for a diatomic molecule, after resumming the formal series for $\mu(h)$ and $w(h)$.

We remark that one can show Proposition 4.4 with the method of HelfferSjöstrand [HS2]. We shall, however, profit from rotational invariance to give a different

Proof of Proposition 4.4. It suffices to prove that there are $\mu_{j} \in \mathbb{R}, b_{j} \in C^{\infty}(\Omega)$ for $j=0,1, \ldots$, with $\mu_{0}, \mu_{1}, \mu_{2}, b_{0}$ given by Theorem 4.2 , such that for all $N \in \mathbb{N}$,

$$
\begin{gathered}
E_{-+}\left(\mu^{N}(h)\right) \beta^{N}=e^{-\psi(x) / h} r_{N}(h), \\
b_{j}(R \cdot x)=b_{j}(x) \quad(R \in S O(3), x \in \Omega),
\end{gathered}
$$

where

$$
\mu^{N}(h)=\sum_{j=0}^{N} \mu_{j} h^{j}, \quad \beta^{N}(x, h)=e^{-\psi(x) / h} \sum_{j=0}^{N-1} b_{j}(x) h^{j} \quad \text { and } \quad r_{N} \in S^{-N-1}(\Omega) .
$$

We shall show (4.16) by induction on $N$, profiting from the fact that $E_{-+}(\lambda)$ maps the class of $S O(3)$-invariant symbols $u \in e^{-\psi(x) / h} S(\Omega)$ into itself. For $N=1$, we choose $\mu_{0}=\lambda_{0}, \mu_{1}=\left.\Delta \psi(x)\right|_{\Gamma_{1}}$ as in (4.3) and $b_{0}$ as the (unique and therefore $S O(3)$ invariant) solution of (4.7). In view of (4.13), a straightforward calculation then gives (4.16) for $N=1$. Assuming (4.16) for $N \in \mathbb{N}$ with $b_{0}, \ldots, b_{N-1} S O(3)$-invariant, we have to determine $\mu_{N+1}$ and $b_{N}$. Denoting by $h^{N+1} r_{N, 1}$ the highest order term in the expansion of $r_{N}(h)$, it clearly is constant on $\Gamma_{1}$ by $S O(3)$-invariance of $r_{N}(h)$, and we can take

$$
\mu_{N+1}=\left.r_{N, 1}(x)\right|_{\Gamma_{1}},
$$

and $b_{N}$ as the (unique and $S O(3)$-invariant) solution of

$$
\left\{\begin{array}{l}
2 \nabla \psi \cdot \nabla b_{N}+\left(\Delta \psi-\mu_{1}\right) b_{N}=-\mu_{N+1} b_{0}-r_{N, 1} \quad \text { in } \Omega \\
\left.b_{N}\right|_{\Gamma_{1}}=0
\end{array} .\right.
$$

Since by telescoping

$$
\begin{aligned}
E_{-}+ & \left(\mu^{N+1}(h)\right) \beta^{N+1}=E_{-+}\left(\mu^{N+1}\right)\left(\beta^{N+1}-\beta^{N}\right)+\left(E_{-+}\left(\mu^{N+1}\right)-E_{-+}\left(\mu^{N}\right)\right) \beta^{N} \\
& +E_{-+}\left(\mu^{N}\right) \beta^{N} \\
= & e^{-\psi(x) / h} h^{N+1}\left\{2 \nabla \psi \cdot \nabla b_{N}+\left(\Delta \psi-\mu_{1}\right) b_{N}+\mu_{N+1} b_{0}+r_{N, 1}\right\} \bmod e^{-\psi / h} S^{-N-2},
\end{aligned}
$$


Eq. (4.16) for $N+1$ follows in view of the choice (4.18) and (4.19) for $\mu_{N+1}$ and $b_{N}$. This proves Proposition 4.4.

Finally, we shall associate the quasimode of Theorem 4.2 with the true eigenvalues and eigenfunctions of $P$. Setting $I=\left(-\infty, \lambda_{0}+h \mu_{1}+h^{2} \mu_{2}+\varepsilon h^{5 / 2}\right]$, we know from the general stability result Proposition 1.5, that if $\varepsilon, h>0$ is sufficiently small, $P$ has exactly 1 eigenvalue in $I$ in the case of a linear or planar molecule and exactly 2 eigenvalues in the case of a non-planar molecule. One then proceeds as in the proof of Theorem 3.2 for the diatomic case to show that $\mu(h)$ constructed in Theorem 4.2 gives an asymptotic expansion of these eigenvalues of $P$.

More precisely, one finds

Theorem 4.5. Let $\chi \in C_{0}^{\infty}(\Omega)$ be an $S O(3)$-invariant cut-off function and $\Pi$ denote the spectral projection of $P$ associated with the interval $I$.

Then, with $w(h)$ given by Theorem 4.2, the function

$$
u(h)=\|\Pi \chi w(h)\|^{-1} \Pi \chi w(h)
$$

verifies for all $\varepsilon>0$,

$$
u(x, y ; h)=c(h) \chi(x) w(x, y ; h)+\mathcal{O}\left(e^{-(1-\varepsilon) \psi(x) / h} h^{\infty}\right) \quad \text { in } H^{2}\left(\mathbb{R}^{3 m} \times \mathbb{R}^{p}\right),
$$

where

$$
c(h)=\|\chi w(h)\|^{-1} \sim \sum c_{j} h^{j}, \quad c_{0} \neq 0 .
$$

Furthermore, for a linear or planar molecule, the unique eigenvalue $E(h)$ of $P$ in I satisfies

$$
E(h)=\mu(h)+\mathcal{O}\left(h^{\infty}\right),
$$

and $u(h)$ is the associated normalized eigenfunction. For a non-planar molecule, the two eigenvalues $E_{1}(h)<E_{2}(h)$ of $P$ in I satisfy

$$
E_{j}(h)=\mu(h)+\mathcal{O}\left(h^{\infty}\right) \quad(j=1,2),
$$

and the associated normalized eigenfunctions are

$$
v_{1}(h)=\frac{1}{\sqrt{2(1+\langle u, \tau u\rangle)}}(1+\tau) u(h), \quad v_{2}(h)=\frac{1}{\sqrt{2(1-\langle u, \tau u\rangle)}}(1-\tau) u(h),
$$

where $\tau$ denotes the reflection in $\mathbb{R}^{3 m} \times \mathbb{R}^{p}$, i.e. $\tau u(x, y)=u(-x,-y)$. Thus the asymptotic form of $v_{j}(h)$ is given by inserting (4.22) into (4.25).

We leave it to the reader to deduce the form (4.25) of the eigenfunctions of $P$ from global $O(3)$-invariance of $P$. The normalizing constant $c(h)$ in (4.22) has an asymptotic expansion in $h$ which can be calculated by the stationary phase method since $w(x, y ; h)$ becomes smooth in $x$ after our standard coordinate change. In fact, letting $\left\{\chi_{j}\right\}$ be a partition of unity subordinate to the cover $\left\{\Omega_{j}\right\}$ of $\bar{\Omega}$, we get using (4.15),

$$
\begin{aligned}
\|\chi w(h)\|^{2} & \sim\left\|\chi \sum_{j} \chi_{j} U_{j}^{-1} E_{j}^{+}(\mu(h)) \beta(h)\right\|^{2} \\
& =\sum_{j, k}\left\langle\chi^{2} \chi_{j} E_{j}^{+}(\mu(h)) \beta(h), \chi_{k} U_{j} U_{k}^{-1} E_{k}^{+}(\mu(h)) \beta(h)\right\rangle,
\end{aligned}
$$


where the formal pseudodifferential operator $U_{j} U_{k}^{-1} E_{k}^{+}(\mu(h))$ coincides with $E_{j}^{+}(\mu(h))$ in $\Omega_{j} \cap \Omega_{k}$ in view of (4.11). Thus

$$
\|\chi w(h)\|^{2} \sim \sum_{j=0}^{\infty} \tilde{c}_{j} h^{j}
$$

where $\tilde{c}_{0} \neq 0$, noting that $b_{0}$ defined by (4.7) is positive in $\Omega$ and using (4.12). It follows that $c(h)$ admits an asymptotic expansion of the same form.

\section{Eigenvalue Splitting}

In view of Proposition 4.1, a non-planar molecule leads to a symmetric double well problem. In this final section we shall estimate the splitting between the two first eigenvalues $E_{1}(h)<E_{2}(h)$ of $P(h)$ for such a molecule, assuming (H6). Letting $\Gamma_{1}, \Gamma_{2}$ be the two disjoint connected components of the potential well $\Gamma$, we denote by $d_{j}(x)=d\left(x, \Gamma_{j}\right)$ the distance of a point $x \in \mathbb{R}^{3 m}$ to $\Gamma_{j}$ in the Agmon metric $\left(\lambda_{1}(x)-\lambda_{0}\right) d x^{2}$ and set

$$
S_{0}=\min _{x}\left(d_{1}(x)+d_{2}(x)\right) .
$$

We assume in addition that $\lambda_{1}(x)$ is a discrete eigenvalue of $Q(x)$ for all $x$ satisfying $d_{1}(x)+d_{2}(x)<S_{0}+\varepsilon_{0}$ for some fixed $\varepsilon_{0}>0$. For $\eta>0$ being sufficiently small, we consider the regions

$$
\Omega_{j}=\left\{x \in \mathbb{R}^{3 m} ; d_{j}(x)<S_{0}-\eta\right\}, \quad j=1,2,
$$

and denote by $P_{j}$ the Dirichlet realization of $P$ in $\Omega_{j} \times \mathbb{R}^{p}$. One then has with $\mu(h)$ as in Theorem 4.2

Proposition 5.1. Choosing $\varepsilon>0$ sufficiently small, there is exactly one eigenvalue $E_{j}^{D}(h)$ of $P_{j}$ in $l=\left(-\infty, \lambda_{0}+h \mu_{1}+h^{2}\left(\mu_{2}+\varepsilon\right)\right)$. It verifies

$$
E_{1}^{D}(h)=E_{2}^{D}(h)=\mu(h)+\mathcal{O}\left(h^{\infty}\right) \quad \text { as } h \downarrow 0,
$$

and the associated normalized eigenfunction $u_{j}^{D}$ of $P_{j}$ satisfies

$$
\left\|\nabla_{x, y}\left(e^{d_{j}(x) / h} u_{j}^{D}\right)\right\|_{L^{2}\left(\Omega_{j} \times \mathbb{R}^{p}\right)}+\left\|e^{d_{j}(x) / h} u_{j}^{D}\right\|_{L^{2}\left(\Omega_{j} \times \mathbb{R}^{p}\right)} \leqq C h^{-N_{0}}
$$

for some $N_{0}>0$.

In view of Theorem 4.2, a proof of this proposition is given by combining hypothesis (H1) with the arguments in Sect. 5 of [HS1] which we shall not reproduce here. Note that (5.2) is an improved version of the energy inequality.

We shall now use the well known method of the interaction matrix to compute the splitting $\Delta E=E_{2}-E_{1}$ in terms of the Dirichlet eigenfunctions $u_{j}^{D}$. We choose $\chi_{j} \in C_{0}^{\infty}\left(\Omega_{j}\right)$ with $\chi_{j}(x)=1$ if $d_{j}(x) \leqq S_{0}-\frac{3}{2} \eta, j=1,2$, and set

$$
v_{j}(x, y)=\chi_{j}(x) u_{j}^{D}(x, y) .
$$

Denoting by $\Pi$ the spectral projection of $P(h)$ on the energy range $\left(-\infty, \lambda_{0}+h \mu_{1}\right.$ $\left.+h^{2}\left(\mu_{2}+\varepsilon\right)\right]$, the functions $\Pi v_{1}, \Pi v_{2}$ form a basis of $\operatorname{Ran} \Pi$ for $h>0$ sufficiently small. Orthonormalizing this basis, $\left.P\right|_{\operatorname{Ran} I I}$ is then represented by the matrix

$$
A=\left[\begin{array}{cc}
E^{D} & 0 \\
0 & E^{D}
\end{array}\right]-\left[\begin{array}{cc}
0 & b \\
\bar{b} & 0
\end{array}\right]+\widetilde{\mathscr{O}}\left(e^{-2 S_{0} / h}\right),
$$


where $E^{D}=E_{1}^{D}=E_{2}^{D}$ and

$$
b=\left(\left\langle\nabla_{x} \chi_{1} \cdot \nabla_{x} u_{1}^{D}, v_{2}\right\rangle+\left\langle v_{1}, \nabla_{x} \chi_{2} \cdot \nabla_{x} u_{2}^{D}\right\rangle+\frac{1}{2}\left\langle\left|\nabla \chi_{1}\right|^{2} u_{1}^{D}, v_{2}\right\rangle+\frac{1}{2}\left\langle v_{1},\left|\nabla \chi_{2}\right|^{2} u_{2}^{D}\right\rangle\right)
$$

(see [HS1] for the calculation). We recall that our notation for the remainder terms means that for any $\varepsilon>0$ there is $\eta>0$ such that the remainder in (5.3) is of order $\mathcal{O}_{\varepsilon}\left(e^{-\left(2 S_{0}-\varepsilon\right) / h}\right)$. The representation $(5.3)$ then leads to

Theorem 5.2. Let $E_{1}<E_{2}$ be the first eigenvalues of $P$. Then

$$
E_{1}=E^{D}-|b|+\widetilde{O}\left(e^{-2 S_{0} / h}\right), \quad E_{2}=E^{D}+|b|+\widetilde{\mathcal{O}}\left(e^{-2 S_{0} / h}\right),
$$

and the splitting $\Delta E=E_{2}-E_{1}$ satisfies

$$
0<\Delta E \leqq C h^{-N_{1}} e^{-S_{0} / h}
$$

for some $N_{1}>0$, if $h>0$ is sufficiently small.

Proof. Equation (5.5) being a trivial consequence of (5.3), we only prove (5.6). Note that (5.2) implies

$$
\left\|e^{d_{j} / h} \nabla_{x} u_{j}^{D}\right\|_{L^{2}\left(\Omega_{j} \times \mathbb{R}^{p}\right)}+\left\|e^{d_{j} / h} u_{j}^{D}\right\|_{L^{2}\left(\Omega_{j} \times \mathbb{R}^{p}\right)} \leqq C h^{-N}
$$

for some $N>0$. In fact

$$
\left\|e^{d_{j} / h} \nabla_{x} u_{j}^{D}\right\| \leqq\left\|\nabla_{x}\left(e^{d_{j} / h} u_{j}^{D}\right)\right\|+h^{-1}\left\|\left(\nabla_{x} d_{j}\right) e^{d_{j} / h} u_{j}^{D}\right\|
$$

and using $\left|\nabla_{x} d_{j}(x)\right|^{2} \leqq \lambda_{1}(x)-\lambda_{0}$ a.e. in $x \in \mathbb{R}^{3 m}$, one gets

$$
\begin{aligned}
\left\|\left(\nabla_{x} d_{j}\right) e^{d_{j} / h} u_{j}^{D}\right\|^{2} & \leqq\left\langle\left(\lambda_{1}(x)-\lambda_{0}\right) e^{d_{j} / h} u_{j}^{D}, e^{d_{j} / h} u_{j}^{D}\right\rangle \\
& \leqq\left\langle\left(Q(x)-\lambda_{0}\right) e^{d_{j} / h} u_{j}^{D}, e^{d_{j} / h} u_{j}^{D}\right\rangle \\
& \leqq 2\left\|\nabla_{x, y}\left(e^{d_{j} / h} u_{j}^{D}\right)\right\|^{2}+C\left\|e^{d_{j} / h} u_{j}^{D}\right\|^{2} .
\end{aligned}
$$

The last estimate follows from hypothesis (H1), which in particular implies that $V(x, y)+W(x)$ is $-\Delta_{x}-\Delta_{y}$-form bounded with relative bound $<1$. Thus $(5.7)$ follows from (5.2) combining (5.8) and (5.9). Since $\Delta E=2|b|+\widetilde{\mathcal{O}}\left(e^{-2 S_{0} / h}\right)$, one gets (5.6) by use of (5.4) and (5.7).

To obtain an asymptotic expansion of the splitting $\Delta E$, we are forced to make additional hypotheses on the geometry of the minimal geodesics connecting the two wells $\Gamma_{1}$ and $\Gamma_{2}$. In particular we have to exclude the possibility that some geodesic meets the set $\mathscr{C}$ of nuclear collisions. Even in the physical case of repulsive Coulomb interactions between the nuclei, this might a priori be possible since $d_{j}(x)$ stays bounded on $\mathscr{C}$. Thus we assume

$$
d_{1}(x)+d_{2}(x)>S_{0}, \quad(x \in \mathscr{C}),
$$

which implies that $\left\{x \in \mathbb{R}^{3 m} ; d_{1}(x)+d_{2}(x) \leqq S_{0}+\varepsilon_{0}\right\}=\mathscr{M}_{\varepsilon_{0}}$ and $\mathscr{C}$ are disjoint for $\varepsilon_{0}>0$ sufficiently small. We choose a region $\Omega \subset C \Omega_{1}$ with $C^{\infty}$-boundary $\partial \Omega$ such that $\Gamma_{1} \subset \Omega$, and set $L=\partial \Omega \cap \mathscr{M}_{\varepsilon_{0}}$. As in [HS1] one computes

$$
b=2 h^{2} \int_{L} \int_{\mathbb{R}^{p}}\left(u_{2}^{D} \partial_{n_{x}} u_{1}^{D}-u_{1}^{D} \partial_{n_{x}} u_{2}^{D}\right) d S_{x} d y+\mathcal{O}\left(e^{-S_{0} / h} h^{\infty}\right) .
$$

To get an asymptotic expansion of $b$ by stationary phase, we assume 
The minimal geodesics connecting $\Gamma_{1}$ and $\Gamma_{2}$ (with endpoints removed) form a 4-dimensional $C^{\infty}$-manifold $M$ which intersects $L$ transversally, and

$$
d_{1}(x)+d_{2}(x) \geqq S_{0}+c_{0} d^{2}(x, L \cap M), \quad(x \in L)
$$

for some $c_{0}>0$.

Theorem 5.2. Assuming (5.10) and (5.12), the splitting $\Delta E=E_{2}-E_{1}$ admits a complete asymptotic expansion

$$
\Delta E \sim e^{-S_{0} / h} h \sum_{j=0}^{\infty} \alpha_{j} h^{j}, \quad \text { with } \quad \alpha_{0} \neq 0 .
$$

Proof. In view of (5.10) there is an open neighborhood of $\Gamma_{j} \cup M, j=1,2$, where $\lambda_{1}(x)$ is a discrete eigenvalue of $Q(x)$ and hence is $C^{\infty}$ in $x$. Thus, according to [HS2], there is an open neighborhood $W_{j}$ of $\Gamma_{j} \cup M$, such that $d_{j} \in C^{\infty}\left(W_{j}\right)$ and $\lambda_{1} \in C^{\infty}\left(W_{1}\right.$ $\cup W_{2}$ ). Applying Theorem 4.2 to each well $\Gamma_{j}$ we obtain approximate eigenfunctions $w_{j}(h)$ near $\Gamma_{j}$, and using the transport equations we can extend $w_{j}(h)$ to $W_{j}$ such that

$$
\begin{gathered}
(P(h)-\mu(h)) w_{j}(h)=\mathcal{O}\left(h^{\infty} e^{-d_{j} / h}\right) \quad \text { in } W_{j}, \\
w_{j}(x, y ; h) \sim e^{-d_{j} / h} h^{-(3 m-3) / 4} \sum_{k=0}^{\infty} a_{j k}(x, y) h^{k},
\end{gathered}
$$

where $a_{j 0}(x, y)=b_{j 0}(x) u_{1}(x, y)$ with $b_{j 0}(x)>0$ in $W_{j}$ and $\left.b_{j 0}\right|_{\Gamma_{j}}=1$. It follows from (4.27) that the normalized approximate eigenfunctions which we still denote by $w_{j}(h)$ admit a similar asymptotic expansion, and in view of (5.2) we find that for each compact set $K \subset W_{j}$,

$$
\left\|e^{d_{J} / h} \nabla_{x, y}\left(u_{j}^{D}-w_{j}\right)\right\|_{L^{2}\left(K \times \mathbb{R}^{p}\right)}+\left\|e^{d_{j} / h}\left(u_{j}^{D}-w_{j}\right)\right\|_{L^{2}\left(K \times \mathbb{R}^{p}\right)}=\mathcal{O}\left(h^{\infty}\right) .
$$

Since $P(h)\left(u_{j}^{D}-w_{j}\right)=\mathcal{O}\left(h^{\infty} e^{-d_{j} / h}\right)$ in $K \times \mathbb{R}^{p}$, one gets from (5.15) that

$$
\sum_{|\beta|+|\alpha|=2}\left\|e^{d_{j} / h}\left(h D_{x}\right)^{\alpha} D_{y}^{\beta}\left(u_{j}^{D}-w_{j}\right)\right\|_{L^{2}\left(K \times \mathbb{R}^{p}\right)}=\mathcal{O}\left(h^{\infty}\right) .
$$

It follows that in (5.11) we may replace $u_{j}^{D}$ by $w_{j}$ to get

$$
b=2 h^{2} \int_{L} \int_{\mathbb{R}^{p}}\left(w_{2} \partial_{n_{x}} w_{1}-w_{1} \partial_{n_{x}} w_{2}\right) d y d S_{x}+\mathcal{O}\left(h^{\infty} e^{-S_{0} / h}\right) .
$$

A priori $w_{j}(x, y)$ is only in $C^{2}\left(W_{j}, L^{2}\left(\mathbb{R}^{p}\right)\right)$. But one can then use our standard coordinate change as in the remark ending Sect. 4 to show that

$$
\int_{\mathbb{R}^{p}}\left(w_{2} \partial_{n_{x}} w_{1}-w_{1} \partial_{n_{x}} w_{2}\right) d y \in C^{\infty}(L)
$$

Hypothesis (5.12) then allows to calculate the asymptotic expansion of $b$ by the stationary phase method. We leave it to the reader to fill in the straightforward details and thus complete the proof of Theorem 5.2.

Finally we remark that dropping assumption (5.12) and keeping assumption (5.10), one can still derive precise upper and lower bounds on the splitting, using the methods of [Ma1]. Although we don't offer a proof, we think that (5.10) is generic since $\lambda_{1}(x)$ tends to infinity as $x$ approaches the set $\mathscr{C}$ of nuclear collisions.

Acknowledgements. The second author would like to thank B. Helffer and L. Robbiano for useful discussions on the subject. X.P. Wang acknowledges the financial support of A. von Humboldt Stiftung. 


\section{References}

[A] Agmon, S.: Lectures on exponential decay of solutions of second-order elliptic equations. Princeton, NJ: Princeton University Press 1982

[AS] Aventini, P., Seiler, R.: On the electronic spectrum of the diatomic molecule. Commun. Math. Phys. 22, 269-279 (1971)

[BK] Balazard-Konlein, A.: Calcul fonctionel pour des opérateur $h$-admissible à symbole opérateurs at applications. Thése de 3ème cycle, Université de Nantes (1985)

[BO] Born, M., Oppenheimer, R.: Zur Quantentheorie der Molekeln. Annal. Phys. 84, 457 (1927)

[BT] Bott, R., Tu, L.W.: Differential forms in algebraic topology. Berlin, Heidelberg, New York: Springer 1982

[CDS] Combes, J.M., Duclos, P., Seiler, R.: The Born-Oppenheimer approximation. In: Rigorous atomic and molecular physics. Velo, G., Wightman, A. (eds.). pp. 185-212. New York: Plenum Press 1981

[CS] Combes, J.M., Seiler, R.: Regularity and asymptotic properties of the discrete spectrum of electronic Hamiltonians. Int. J. Quant. Chem. XIV, 213-229 (1978)

[GMS] Gerard, C., Martinez, A., Sjöstrand, J.: A mathematical approach to the effective Hamiltonian in perturbed periodic problems. Preprint Orsay 1990, submitted to Commun. Math. Phys.

[HS1] Helffer, B., Sjöstrand, J.: Multiple wells in the semiclassical limit I. Commun. Partial Differ. Equations 9 (4), 337-408 (1984)

[HS2] Helffer, B., Sjöstrand, J.: Puits multiples en mécanique semi-classique VI (Cas des puits sous-variétés). Ann. Inst. Henri Poincaré 46, 353-372 (1987)

[Ha1] Hagedorn, G.A.: High order corrections to the time-independent Born-Oppenheimer approximation. I. Smooth potentials. Ann. Inst. Henri Poincaré 47, 1-16 (1987)

[Ha2] Hagedorn, G.A.: High order corrections to the time-independent Born-Oppenheimer approximation. II. Diatomic Coulomb systems. Commun. Math. Phys. 116, 23-44 (1988)

[Hu] Hunziker, W.: Distortion analycity and molecular resonance curves. Ann. Inst. H. Poincaré 45, 339-358 (1986)

[Hus] Husemoller, D.: Fiber bundles. Berlin, Heidelberg, New York: Springer 1975

[K] Klein, M.: On the mathematical theory of predissociation. Ann. Phys. 178, 48-73 (1987)

[Ma1] Martinez, A.: Estimations de l'effet tunnel pour le double puits I. J. Math. Pures Appl. 66, 195-215 (1987)

[Ma2] Martinez, A.: Développements asymptotiques et effet tunnel dans l'approximation de Born-Oppenheimer. Ann. Inst. Henri Poincaré 49, 239-257 (1989)

[Ma3] Martinez, A.: Resonances dans l'approximation de Born-Oppenheimer I. J. Diff. Eq. (to appear)

[Ma4] Martinez, A.: Resonances dans l'approximation de Born-Oppenheimer II - Largeur des resonances. Commun. Math. Phys. 135, 517-530 (1991)

[S] Seiler, R.: Does the Born-Oppenheimer approximation work? Helv. Phys. Acta 46, 230-234 (1973)

Communicated by T. Spencer 
